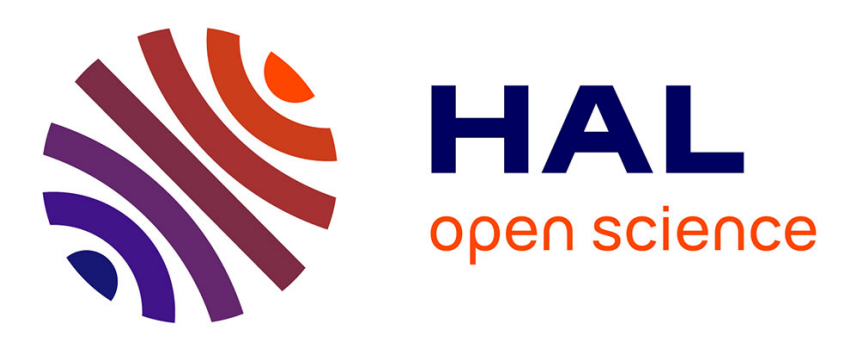

\title{
On the approximation of electromagnetic fields by edge finite elements. Part 3: sensitivity to coefficients Patrick Ciarlet
}

\section{To cite this version:}

Patrick Ciarlet. On the approximation of electromagnetic fields by edge finite elements. Part 3: sensitivity to coefficients. SIAM Journal on Mathematical Analysis, 2020, 10.1137/19M1275383 . hal-02276430v3

\section{HAL Id: hal-02276430 \\ https://hal.inria.fr/hal-02276430v3}

Submitted on 17 Jun 2020

HAL is a multi-disciplinary open access archive for the deposit and dissemination of scientific research documents, whether they are published or not. The documents may come from teaching and research institutions in France or abroad, or from public or private research centers.
L'archive ouverte pluridisciplinaire HAL, est destinée au dépôt et à la diffusion de documents scientifiques de niveau recherche, publiés ou non, émanant des établissements d'enseignement et de recherche français ou étrangers, des laboratoires publics ou privés. 


\title{
ON THE APPROXIMATION OF ELECTROMAGNETIC FIELDS BY EDGE FINITE ELEMENTS. PART 3: SENSITIVITY TO COEFFICIENTS
}

\author{
PATRICK CIARLET, JR.*
}

\begin{abstract}
In bounded domains, the regularity of the solutions to boundary value problems depends on the geometry, and on the coefficients that enter into the definition of the model. This is in particular the case for the time-harmonic Maxwell equations, whose solutions are the electromagnetic fields. In this paper, emphasis is put on the electric field. We study the regularity in terms of the fractional order Sobolev spaces $H^{s}, s \in[0,1]$. Precisely, our first goal is to determine the regularity of the electric field and of its curl, that is to find some regularity exponent $\tau \in(0,1)$, such that they both belong to $H^{s}$, for all $s \in[0, \tau)$. After that, one can derive error estimates. Here, the error is defined as the difference between the exact field and its approximation, where the latter is built with Nédélec's first family of finite elements. In addition to the regularity exponent, one needs to derive a stability constant that relates the norm of the error to the norm of the data: this is our second goal. We provide explicit expressions for both the regularity exponent and the stability constant with respect to the coefficients. We also discuss the accuracy of these expressions, and we provide some numerical illustrations.
\end{abstract}

Key words. Maxwell's equations, interface problem, edge elements, sensitivity to coefficients, error estimates

AMS subject classifications. 78A48, 35B65, 65N30

1. Introduction. We study the numerical approximation of electromagnetic fields governed by Maxwell's equations. More precisely, our goal is to characterize the dependence of the error between the exact and computed fields, with respect to the coefficients that define the model (PDEs, supplemented with boundary conditions). This paper is the third part of the series entitled "On the Approximation of Electromagnetic Fields by Edge Finite Elements" [12, 13].

For Maxwell's equations, the coefficients are the electric permittivity, the magnetic permeability and the conductivity. Classically, the model is recast as an equivalent variational formulation. The first goal is to determine the value of the constants that appear in the analysis of the variational formulation, which are the continuity modulus of the forms, and the coercivity or inf-sup constants. Then, one performs the numerical analysis of the model. In addition to the above-mentioned constants, one has to estimate the order of convergence, which depends on the (extra-)regularity of the fields ; this (extra-)regularity depends itself on the behavior of the coefficients, and on the geometry of the model. In particular, it is crucial to use ad hoc norms to measure the fields and the data, and particular care is devoted to the definition of those norms. We observe that if the coefficients belong to a set not reduced to a singleton (eg. random coefficients), then the (extra-)regularity may vanish in some limit cases.

The outline of the paper is as follows.

In the next section, we introduce the model problem (see eg. [2]), set in a bounded region of $\mathbb{R}^{3}$, with volume sources. We prescribe some a priori conditions on the coefficients, and on the source terms; the coefficients are only supposed to be piecewise smooth, hence they may be discontinuous. The variational formulation is introduced.

*POEMS, ENSTA Paris, Institut Polytechnique de Paris, 828 Bd des Maréchaux, 91762 Palaiseau Cedex, France (patrick.ciarlet@ensta-paris.fr). 
Then, in section 3, we recall the main results regarding the discretisation of electromagnetic fields by Nédélec's finite elements [30, 29, 12, 20]. We also define the function spaces that will allow us to perform the analysis of the model. Particular attention is paid to the fractional order Sobolev spaces, which play a crucial role in the analysis. These are defined using either the real interpolation method [28, 33, 9], or with the help of the Sobolev-Slobodeckij norms [24, 25, 11]: this yields two ways to measure their elements. In section 4, we provide the (well-known) estimates for the continuity modulus and the coercivity constant related to the variational formulation, with respect to the coefficients of the model.

There remains to estimate the so-called regularity exponent and the stability constant, which relate the norm of the electromagnetic fields in ad hoc fractional order Sobolev spaces norms to the norm of the source terms. Estimating these last two quantities with respect to the coefficients of the model is less classical. Hence, most novelties in the paper are contained in the approach developed in sections 5 and 6 . In section 5 , we recall how one can split the electromagnetic fields into a regular part and a gradient part [4], so the (lack of) regularity of the fields rests on the regularity of the gradients. This is the subject of section 6 . We use a perturbation argument à la Jochmann [27] or Bonito et al [6] to estimate this regularity with respect to the coefficients of the model. We call it the global approach. When the coefficient are piecewise constant, one may also use the local approach, see Appendix B. The main novelties are threefold: the extension of existing results to problems with complex-valued coefficients, set in a non-topologically trivial domain; the use of the two measures for elements of the Sobolev spaces, and their interplay; the design of estimates for the numerical error that depend only on the coefficients of the model (see Theorem 6.15). To conclude, we illustrate our results by two examples in section 7 .

We denote constant fields by the symbol $c s t$. Vector-valued (respectively tensorvalued) function spaces are written in boldface character (resp. blackboard bold characters). Given a non-empty open set $\mathcal{O}$ of $\mathbb{R}^{3}$, we use the notation $(\cdot \cdot \cdot)_{0, \mathcal{O}}$ (respectively $\left.\|\cdot\|_{0, \mathcal{O}}\right)$ for the $L^{2}(\mathcal{O})$ and the $\boldsymbol{L}^{2}(\mathcal{O}):=\left(L^{2}(\mathcal{O})\right)^{3}$ hermitian scalar products (resp. norms). More generally, $(\cdot \mid \cdot)_{s, \mathcal{O}}$ and $\|\cdot\|_{s, \mathcal{O}}$ (respectively $|\cdot|_{s, \mathcal{O}}$ ) denote the hermitian scalar product and the norm (resp. semi-norm) of the Sobolev spaces $H^{s}(\mathcal{O})$ and $\boldsymbol{H}^{s}(\mathcal{O}):=\left(H^{s}(\mathcal{O})\right)^{3}$ for $s \in \mathbb{R}$ (resp. for $s>0$ ). The index zmv indicates zero-mean-value fields. If moreover the boundary $\partial \mathcal{O}$ is Lipschitz, $\boldsymbol{n}$ denotes the unit outward normal vector field to $\partial \mathcal{O}$. Finally, it is assumed that the reader is familiar with function spaces related to Maxwell's equations, such as $\boldsymbol{H}(\mathbf{c u r l} ; \mathcal{O})$, $\boldsymbol{H}_{0}(\boldsymbol{\operatorname { c u r l }} ; \mathcal{O}), \boldsymbol{H}(\operatorname{div} ; \mathcal{O}), \boldsymbol{H}_{0}(\operatorname{div} ; \mathcal{O})$ etc. A priori, $\boldsymbol{H}(\mathbf{c u r l} ; \mathcal{O})$ is endowed with the norm $\boldsymbol{v} \mapsto\left(\|\boldsymbol{v}\|_{0, \mathcal{O}}^{2}+\|\operatorname{curl} \boldsymbol{v}\|_{0, \mathcal{O}}^{2}\right)^{1 / 2}$, etc. We refer to the monographs of Monk [29] and Assous et al [4] for details. We will define more specialized function spaces later on.

2. The model problem. Let $\Omega$ be a domain in $\mathbb{R}^{3}$, that is an open, connected and bounded subset of $\mathbb{R}^{3}$ with a Lipschitz-continuous boundary $\partial \Omega$. We are interested in solving the time-harmonic Maxwell's equations (with time-dependence $\exp (-\imath \omega t)$, for a given pulsation $\omega>0$ ),

$$
\begin{aligned}
& \imath \omega \boldsymbol{d}+\operatorname{curl} \boldsymbol{h}=\boldsymbol{j} \text { in } \Omega, \\
& -\imath \omega \boldsymbol{b}+\operatorname{curl} \boldsymbol{e}=0 \text { in } \Omega, \\
& \operatorname{div} \boldsymbol{d}=\varrho \text { in } \Omega, \\
& \operatorname{div} \boldsymbol{b}=0 \text { in } \Omega .
\end{aligned}
$$


Above, $(\boldsymbol{e}, \boldsymbol{d}, \boldsymbol{h}, \boldsymbol{b})$ are the electromagnetic fields. We suppose that $\boldsymbol{d}$ and $\boldsymbol{b}$ are related to $\boldsymbol{e}$ and $\boldsymbol{h}$ by the constitutive relations

$$
\boldsymbol{d}=\varepsilon \boldsymbol{e}, \boldsymbol{b}=\mu \boldsymbol{h} \text { in } \Omega,
$$

where the real-valued coefficient $\varepsilon$ is the electric permittivity and the real-valued coefficient $\mu$ is the magnetic permeability.

The source terms $\boldsymbol{j}$ and $\varrho$ are respectively the current density and the charge density, and they are related by the charge conservation equation

$$
-\imath \omega \varrho+\operatorname{div} \boldsymbol{j}=0 .
$$

We suppose that the current density may be written as

$$
\boldsymbol{j}=\boldsymbol{j}_{\text {ext }}+\sigma \boldsymbol{e} \text { in } \Omega,
$$

where $\boldsymbol{j}_{\text {ext }}$ is an externally imposed current, and the real-valued coefficient $\sigma$ is the conductivity.

2.1. A priori assumptions. Classically, the electromagnetic fields all belong to $\boldsymbol{L}^{2}(\Omega)$ and the coefficients $\varepsilon, \mu$ and $\sigma$ have a fixed-sign (positive): we make these assumptions from now on. We also assume throughout this work that these coefficients together with their inverses belong to $L^{\infty}(\Omega)$, and we use the notations $\varepsilon_{\max }=$ $\|\varepsilon\|_{L^{\infty}(\Omega)}, \varepsilon_{\min }=\left(\left\|\varepsilon^{-1}\right\|_{L^{\infty}(\Omega)}\right)^{-1}$, etc.

We choose the data $\left(\boldsymbol{j}_{\text {ext }}, \varrho\right)$ in $\boldsymbol{H}(\operatorname{div} ; \Omega) \times H^{-1}(\Omega)$. It is also possible to choose $\boldsymbol{j}_{\text {ext }} \in \boldsymbol{L}^{2}(\Omega)$ with $\operatorname{div} \boldsymbol{j}_{\text {ext }} \in H^{-t}(\Omega)$ for some $t \in(0,1)$, but we assume for simplicity that $\operatorname{div} \boldsymbol{j}_{\text {ext }} \in L^{2}(\Omega)$. We refer to $\S 6.5$ for the study of the more general case.

Finally, we assume that the medium $\Omega$ is surrounded by a perfect conductor, so that the boundary condition below holds:

$$
\boldsymbol{e} \times \boldsymbol{n}=0 \quad \text { on } \partial \Omega .
$$

Eqs. (2.1)-(2.8) together with the assumptions on the coefficients and on the source terms define our model problem. When we focus on the discretization, see $\S 3.3$ and afterwards, we assume that $\Omega$ is a Lipschitz polyhedron, that $\varepsilon, \sigma$ are piecewise smooth on $\Omega$, and that $\mu$ is constant on $\Omega$. We call this setting the polyhedral model problem. Let us mention that once the field $\boldsymbol{e}$ is known, then all other electromagnetic fields $\boldsymbol{d}$, $\boldsymbol{b}$ and $\boldsymbol{h}$ are known too. As a consequence, we focus on the study of the field $\boldsymbol{e}$. In particular, we note that $\boldsymbol{e}$ belongs to the function space $\boldsymbol{H}_{0}(\mathbf{c u r l} ; \Omega)$.

2.2. Variational formulation. In the spirit of the charge conservation equation, let us introduce $\varrho_{e x t}=-\imath / \omega \operatorname{div} \boldsymbol{j}_{\text {ext }} \in L^{2}(\Omega)$. Our model problem can be formulated in the electric field $e$ only, namely

$$
\left\{\begin{array}{l}
\text { Find } \boldsymbol{e} \in \boldsymbol{H}_{0}(\operatorname{curl} ; \Omega) \text { such that } \\
-\omega^{2} \varepsilon_{\sigma} \boldsymbol{e}+\operatorname{curl}\left(\mu^{-1} \operatorname{curl} \boldsymbol{e}\right)=\imath \omega \boldsymbol{j}_{\text {ext }} \text { in } \Omega \\
\operatorname{div} \varepsilon_{\sigma} \boldsymbol{e}=\varrho_{\text {ext }} \text { in } \Omega .
\end{array}\right.
$$

Above, the complex-valued coefficient $\varepsilon_{\sigma}$ is defined by $\varepsilon_{\sigma}=\varepsilon+\imath \sigma / \omega$. Note that in (2.9), the equation $\operatorname{div} \varepsilon_{\sigma} \boldsymbol{e}=\varrho_{\text {ext }}$ is implied by the second-order equation $-\omega^{2} \varepsilon_{\sigma} \boldsymbol{e}+$ $\operatorname{curl}\left(\mu^{-1} \operatorname{curl} \boldsymbol{e}\right)=\imath \omega \boldsymbol{j}_{\text {ext }}$, together with the charge conservation equation (2.6) and the splitting of the current (2.7), so it can be omitted. Moreover, one can check that the equivalent variational formulation in $\boldsymbol{H}_{0}(\mathbf{c u r l} ; \Omega)$ writes

$(2.10)\left\{\begin{array}{l}\text { Find } \boldsymbol{e} \in \boldsymbol{H}_{0}(\operatorname{curl} ; \Omega) \text { such that } \\ \left(\mu^{-1} \operatorname{curl} \boldsymbol{e} \mid \operatorname{curl} \boldsymbol{v}\right)_{0, \Omega}-\omega^{2}\left(\varepsilon_{\sigma} \boldsymbol{e} \mid \boldsymbol{v}\right)_{0, \Omega}=\imath \omega\left(\boldsymbol{j}_{\text {ext }} \mid \boldsymbol{v}\right)_{0, \Omega}, \forall \boldsymbol{v} \in \boldsymbol{H}_{0}(\operatorname{curl} ; \Omega) .\end{array}\right.$ 
Under the assumptions on the coefficients, this variational formulation is well-posed (see for instance $[4, \S 8.3 .2]$ ). In other words,

$$
\begin{aligned}
& \exists C_{(\varepsilon, \mu, \sigma)}>0, \text { such that } \forall \boldsymbol{j}_{\text {ext }} \in \boldsymbol{L}^{2}(\Omega), \exists ! \boldsymbol{e} \text { solution to }(2.10) \text {, and } \\
& \|\boldsymbol{e}\|_{\boldsymbol{H}(\mathbf{c u r l} ; \Omega)} \leq C_{(\varepsilon, \mu, \sigma)}\left\|\boldsymbol{j}_{\text {ext }}\right\|_{0, \Omega} \text {. }
\end{aligned}
$$

3. Discretisation of electromagnetic fields. Here, we define finite element approximations of the electric field $\boldsymbol{e}$. We also recall how one can build an a priori error estimate between $\boldsymbol{e}$ and its approximation. When we study the numerical approximations and for the ease of exposition, we assume that $\Omega$ is a Lipschitz polyhedron (polyhedral model problem). To define finite dimensional subspaces $\left(\boldsymbol{V}_{h}\right)_{h}$ of $\boldsymbol{H}_{0}(\mathbf{c u r l} ; \Omega)$, we choose the so-called Nédélec's first family of edge finite elements, defined on simplicial meshes of $\Omega$. We follow here [12, §2.4]. It is sufficient to use first-order finite elements because we focus on electromagnetic fields with low regularity. $\bar{\Omega}$ is triangulated by a shape regular family of meshes $\left(\mathcal{T}_{h}\right)_{h}$, made up of (closed) simplices, generically denoted by $K$. A mesh is indexed by $h:=\max _{K} h_{K}$ (the meshsize), where $h_{K}$ is the diameter of $K$. Denoting by $\rho_{K}$ the diameter of the largest ball inscribed in $K$, we assume that there exists a shape regularity parameter $\varsigma>0$ such that for all $h$, for all $K \in \mathcal{T}_{h}$, it holds $h_{K} \leq \varsigma \rho_{K}$. Nédélec's $\boldsymbol{H}$ (curl; $\Omega$ )-conforming (first family, first-order) finite element spaces are defined as

$$
\boldsymbol{V}_{h}:=\left\{\boldsymbol{v}_{h} \in \boldsymbol{H}_{0}(\operatorname{curl} ; \Omega): \boldsymbol{v}_{h \mid K} \in \mathcal{R}_{1}(K), \forall K \in \mathcal{T}_{h}\right\}
$$

where $\mathcal{R}_{1}(K)$ is the six-dimensional vector space of polynomials on $K$

$$
\mathcal{R}_{1}(K):=\left\{\boldsymbol{v} \in \boldsymbol{P}_{1}(K): \boldsymbol{v}(\boldsymbol{x})=\boldsymbol{a}+\boldsymbol{b} \times \boldsymbol{x}, \boldsymbol{a}, \boldsymbol{b} \in \mathbb{R}^{3}\right\} .
$$

According to [30, Theorem 1], any element $\boldsymbol{v}$ in $\mathcal{R}_{1}(K)$ is uniquely determined by the degrees of freedom in the moment set $M_{E}(\boldsymbol{v})$ :

$$
M_{E}(\boldsymbol{v}):=\left(\int_{e} \boldsymbol{v} \cdot \boldsymbol{t} d l\right)_{e \in A_{K}} .
$$

Above, $A_{K}$ is the set of edges of $K$, and $\boldsymbol{t}$ is a unit vector along the edge $e$. The global set of moments on $\boldsymbol{V}_{h}$ is then obtained by taking one degree of freedom as above per interior edge of $\mathcal{T}_{h}$. We recall that the basic approximability property writes (cf. [29, Lemma 7.10])

$$
\lim _{h \rightarrow 0}\left(\inf _{\boldsymbol{v}_{h} \in \boldsymbol{V} h}\left\|\boldsymbol{v}-\boldsymbol{v}_{h}\right\|_{\boldsymbol{H}(\operatorname{curl} ; \Omega)}\right)=0, \forall \boldsymbol{v} \in \boldsymbol{H}_{0}(\operatorname{curl} ; \Omega) .
$$

Assuming for simplicity that the integrals are computed exactly, the discrete electric problem writes

$$
\left\{\begin{array}{l}
\text { Find } \boldsymbol{e}_{h} \in \boldsymbol{V}_{h} \text { such that } \\
\left(\mu^{-1} \operatorname{curl} \boldsymbol{e}_{h} \mid \operatorname{curl} \boldsymbol{v}_{h}\right)_{0, \Omega}-\omega^{2}\left(\varepsilon_{\sigma} \boldsymbol{e}_{h} \mid \boldsymbol{v}_{h}\right)_{0, \Omega}=\imath \omega\left(\boldsymbol{j}_{\text {ext }} \mid \boldsymbol{v}_{h}\right)_{0, \Omega}, \forall \boldsymbol{v}_{h} \in \boldsymbol{V}_{h} .
\end{array}\right.
$$

Because the exact problem is well-posed, cf. (2.11), one may apply Céa's lemma to find

$$
\exists C_{(\varepsilon, \mu, \sigma)}^{\sharp}>0, \forall h,\left\|\boldsymbol{e}-\boldsymbol{e}_{h}\right\|_{\boldsymbol{H}(\operatorname{curl} ; \Omega)} \leq C_{(\varepsilon, \mu, \sigma)}^{\sharp} \inf _{\boldsymbol{v}_{h} \in \boldsymbol{V}_{h}}\left\|\boldsymbol{e}-\boldsymbol{v}_{h}\right\|_{\boldsymbol{H}(\operatorname{curl} ; \Omega)} .
$$


Classically, the constant $C_{(\varepsilon, \mu, \sigma)}^{\sharp}$ depends on the coercivity constant and on the norm of the sesquilinear form in the left-hand side of (2.10) and (3.2). This constant is investigated in detail in section 4 . It follows from (3.1) that

$$
\lim _{h \rightarrow 0}\left\|\boldsymbol{e}-\boldsymbol{e}_{h}\right\|_{\boldsymbol{H}(\operatorname{curl} ; \Omega)}=0 .
$$

In order to obtain a result which is more accurate, typically a convergence rate in the order of $h^{s}$ for some $s>0$, one has to use information on the (extra-)regularity of the electric field. Let us recall how this can be achieved.

3.1. A few reminders about Sobolev spaces. Let $\mathcal{O} \subset \Omega$ be a non-empty connected open subset of $\mathbb{R}^{3}$ with Lipschitz boundary. To give a precise meaning to the regularity of a scalar or vector field on $\mathcal{O}$, we use the well-known Sobolev scale $\left(H^{s}(\mathcal{O})\right)_{s}$.

(0) For $s \in \mathbb{N}$, one uses the standard definition:

$$
H^{s}(\mathcal{O}):=\left\{v \in L^{2}(\mathcal{O}) \text { s.t. } \forall \alpha \in \mathbb{N}^{3},|\alpha| \leq s, \partial_{\alpha} v \in L^{2}(\mathcal{O})\right\},
$$

equipped with the norm $\|v\|_{s, \mathcal{O}}:=\left(\sum_{\alpha \in \mathbb{N}^{3},|\alpha| \leq s}\left\|\partial_{\alpha} v\right\|_{L^{2}(\mathcal{O})}^{2}\right)^{1 / 2}$. Obviously, $H^{0}(\mathcal{O})=$ $L^{2}(\mathcal{O})$.

(1) To define those spaces for $s>0, s \notin \mathbb{N}$, several possibilities exist. Let us begin with the real interpolation method [28] (see also Appendix A), which allows us to define those Hilbert spaces for non-integer indices $s=m+\sigma, m \in \mathbb{N}, \sigma \in(0,1)$, as

$$
H^{s}(\mathcal{O}):=\left(H^{m}(\mathcal{O}), H^{m+1}(\mathcal{O})\right)_{\sigma, 2} .
$$

The corresponding norm is denoted by $\|\cdot\|_{s, \mathcal{O}}$. In particular, for all $0 \leq s \leq t$, it holds that $H^{t}(\mathcal{O}) \subset H^{s}(\mathcal{O})$ with continuous embedding $[9, \S 14]$ :

$$
\exists C_{(s, t)}>0, \forall v \in H^{t}(\mathcal{O}), \quad\|v\|_{s, \mathcal{O}} \leq C_{(s, t)}\|v\|_{t, \mathcal{O}} .
$$

Given $0<s_{0} \leq s_{1}<t<1, s \mapsto C_{(s, t)}$ is continuous on $\left[s_{0}, s_{1}\right]$.

A well-known alternative is to define, for $\sigma \in(0,1)$ :

$$
\underline{H}^{\sigma}(\mathcal{O}):=\left\{v \in L^{2}(\mathcal{O}) \text { s.t. }|v|_{H^{\sigma}(\mathcal{O})}<\infty\right\},
$$

where

$|v|_{\underline{H}^{\sigma}(\mathcal{O})}:=\left(\int_{\mathcal{O}} \int_{\mathcal{O}} \frac{|v(\boldsymbol{x})-v(\boldsymbol{y})|^{2}}{|\boldsymbol{x}-\boldsymbol{y}|^{3+2 \sigma}} d \boldsymbol{x} d \boldsymbol{y}\right)^{1 / 2}$ is the Sobolev-Slobodeckij semi-norm, and $\underline{H}^{\sigma}(\mathcal{O})$ is endowed with the Sobolev-Slobodeckij norm

$$
\|v\|_{\underline{H}^{\sigma}(\mathcal{O})}:=\left(\|v\|_{0, \mathcal{O}}^{2}+|v|_{\underline{H}^{\sigma}(\mathcal{O})}^{2}\right)^{1 / 2} .
$$

And then, for $s=m+\sigma, m \in \mathbb{N}, \sigma \in(0,1)$ :

$$
\underline{H}^{s}(\mathcal{O}):=\left\{v \in H^{m}(\mathcal{O}) \text { s.t. } \forall \alpha \in \mathbb{N}^{3} \text { with }|\alpha|=m, \partial_{\alpha} v \in \underline{H}^{\sigma}(\mathcal{O})\right\},
$$

endowed with the Sobolev-Slobodeckij norm

$$
\|v\|_{\underline{H}^{s}(\mathcal{O})}:=\left(\|v\|_{m, \mathcal{O}}^{2}+\sum_{\alpha \in \mathbb{N}^{3},|\alpha|=m}\left|\partial_{\alpha} v\right|_{\underline{H}^{\sigma}(\mathcal{O})}^{2}\right)^{1 / 2} .
$$


The Sobolev-Slobodeckij semi-norm is

$$
|v|_{\underline{H}^{s}(\mathcal{O})}:=\left(\sum_{\alpha \in \mathbb{N}^{3},|\alpha|=m}\left|\partial_{\alpha} v\right|_{\underline{H}^{\sigma}(\mathcal{O})}^{2}\right)^{1 / 2} .
$$

For all $s \in \mathbb{R}^{+} \backslash \mathbb{N}$, it holds that $H^{s}(\mathcal{O})=\underline{H}^{s}(\mathcal{O})$ algebraically and topologically:

$$
\exists m_{(s)}, M_{(s)}>0, \forall v \in H^{s}(\mathcal{O}), \quad m_{(s)}\|v\|_{\underline{H}^{s}(\mathcal{O})} \leq\|v\|_{s, \mathcal{O}} \leq M_{(s)}\|v\|_{\underline{H}^{s}(\mathcal{O})} .
$$

However, in a bounded set $\mathcal{O}$, there are no results on the uniform equivalence of Sobolev-Slobodeckij norms and real interpolation norms when $s$ spans $(0,1)$, ie. on bounding one norm with the other times a constant that is independent of $s \in(0,1)$. We refer to $[25,11]$ for illuminating discussions on this topic. On the other hand (see $[24]$ or $[9, \S 14])$, if $s$ spans $\left[s_{0}, s_{1}\right]$ with $0<s_{0} \leq s_{1}<1$, there is a uniform equivalence of norms: in other words, $m, m^{-1}, M, M^{-1}$ are continuous on $\left[s_{0}, s_{1}\right]$.

(2) For $s \geq 0, H_{0}^{s}(\mathcal{O})$ is the closure of $\mathcal{D}(\mathcal{O})$ in $H^{s}(\mathcal{O})$. For $s \in\left[0, \frac{1}{2}\right]$, it holds that $H_{0}^{s}(\mathcal{O})=H^{s}(\mathcal{O})$ algebraically and topologically, see for instance [21, Theorem 1.4.2.4] ; while for $s>\frac{1}{2}$, it holds that $H_{0}^{s}(\mathcal{O}) \subsetneq H^{s}(\mathcal{O})$.

(3) For $s<0, H^{s}(\mathcal{O})$ is the topological dual of $H_{0}^{-s}(\mathcal{O})$.

(4) For $s \geq 0, \widetilde{H}^{s}(\mathcal{O})$ (also denoted in the literature by $H_{00}^{s}(\mathcal{O})$ ) is composed of elements of $H^{s}(\mathcal{O})$ such that the continuation by zero outside $\mathcal{O}$ belongs to $H^{s}\left(\mathbb{R}^{3}\right)$; for $s \notin \frac{1}{2}+\mathbb{N}$, it holds that $\widetilde{H}^{s}(\mathcal{O})=H_{0}^{s}(\mathcal{O})$, while for $s \in \frac{1}{2}+\mathbb{N}$, it holds that $\widetilde{H}^{s}(\mathcal{O}) \subsetneq H_{0}^{s}(\mathcal{O})$. Going back to the real interpolation method, for non-integer indices $s=m+\sigma, m \in \mathbb{N}, \sigma \in(0,1)$, one has $\left.\widetilde{H}^{s}(\mathcal{O})=\left(H_{0}^{m}(\mathcal{O}), H_{0}^{m+1} \mathcal{O}\right)\right)_{\sigma, 2}$.

For the regularity studies, we choose the real interpolation method, while we use the double-integral Sobolev-Slobodeckij norms and semi-norms to perform the numerical analysis, and derive convergence rates.

3.2. Piecewise smooth fields. The set $\mathcal{P}:=\left\{\Omega_{j}\right\}_{j=1, \cdots, J}$ is called a partition of $\Omega$ if $\left(\Omega_{j}\right)_{j=1, \cdots, J}$ are disjoint domains, and it holds $\bar{\Omega}=\cup_{j=1}^{J} \overline{\Omega_{j}}$. When the $\left(\Omega_{j}\right)_{j=1, \cdots, J}$ are Lipschitz polyhedra, we us the term polyhedral partition. Given a partition, we introduce the corresponding interface $\Sigma:=\cup_{1 \leq j \neq j^{\prime} \leq J}\left(\partial \Omega_{j} \cap \partial \Omega_{j^{\prime}}\right)$. For a field $v$ defined on $\Omega$, we denote by $v_{j}$ its restriction to $\Omega_{j}$, for all $j$. In relation to the partition $\mathcal{P}$ and for $s \geq 0$, we define

$$
\begin{aligned}
& P H^{s}(\Omega):=\left\{v \in L^{2}(\Omega): v_{j} \in H^{s}\left(\Omega_{j}\right), 1 \leq j \leq J\right\}, \text { endowed with } \\
& \|v\|_{P H^{s}(\Omega)}:=\left(\sum_{1 \leq j \leq J}\left\|v_{j}\right\|_{s, \Omega_{j}}^{2}\right)^{1 / 2} \text { or }\|v\|_{\underline{P H^{s}}(\Omega)}:=\left(\sum_{1 \leq j \leq J}\left\|v_{j}\right\|_{\underline{H}^{s}\left(\Omega_{j}\right)}^{2}\right)^{1 / 2} .
\end{aligned}
$$

To simplify the notations, the reference to $\mathcal{P}$ is usually omitted. Let us recall the technical result (Theorem 4.1 of [1], or Lemma 2.1 of [6]).

Proposition 3.1. For all $s \in[0,1]$, it holds that

$$
\|v\|_{P H^{s}(\Omega)} \leq\|v\|_{s, \Omega}, \quad \forall v \in H^{s}(\Omega) .
$$

Note that one has $P H^{s}(\Omega)=H^{s}(\Omega)$ algebraically and topologically for all partitions and for all $s \in\left[0, \frac{1}{2}\right)$. 
Finally, we introduce

$$
\begin{aligned}
& \boldsymbol{P} \boldsymbol{H}^{s}(\operatorname{curl} ; \Omega):=\left\{\boldsymbol{v} \in \boldsymbol{P H}^{s}(\Omega): \operatorname{curl} \boldsymbol{v} \in \boldsymbol{P} \boldsymbol{H}^{s}(\Omega)\right\} \text { for } s>0 \\
& P W^{1, \infty}(\Omega):=\left\{\zeta \in L^{\infty}(\Omega): \zeta_{j} \in W^{1, \infty}\left(\Omega_{j}\right), 1 \leq j \leq J\right\} .
\end{aligned}
$$

$\boldsymbol{P} \boldsymbol{H}^{s}(\mathbf{c u r l} ; \Omega)$ is endowed with the graph norm. We observe that one has the embedding $\boldsymbol{P} \boldsymbol{H}^{s}(\mathbf{c u r l} ; \Omega) \subset \boldsymbol{H}(\mathbf{c u r l} ; \Omega)$, according to the definition of $\boldsymbol{P} \boldsymbol{H}^{s}(\Omega)$.

And we endow $P W^{1, \infty}(\Omega)$ with the norm $\|\zeta\|_{P W^{1, \infty}(\Omega)}:=\|\zeta\|_{L^{\infty}(\Omega)}+|\zeta|_{P W^{1, \infty}(\Omega)}$, and the semi-norm $|\zeta|_{P W^{1, \infty}(\Omega)}:=\max _{1 \leq j \leq J}\left\|\nabla \zeta_{j}\right\|_{L^{\infty}\left(\Omega_{j}\right)}$. For a piecewise constant coefficient $\zeta$, it holds that $\|\zeta\|_{P W^{1, \infty}(\Omega)}=\|\zeta\|_{L^{\infty}(\Omega)}=\max _{1 \leq j \leq J}\left|\zeta_{j}\right|$.

When the partition is trivial, that is $\mathcal{P}=\{\Omega\}$, we omit the $P$ or $\boldsymbol{P}$ in the name of the function space.

We note that, for the polyhedral model, the assumption on the coefficients writes $\varepsilon, \sigma \in P W^{1, \infty}(\Omega)$, and the interface $\Sigma$ can be viewed as the locus of the discontinuities of at least one the two coefficients. More generally, if $\varepsilon, \sigma, \mu \in P W^{1, \infty}(\Omega), \Sigma$ is the locus of the discontinuities of at least one the three coefficients.

3.3. Finite element interpolation or quasi-interpolation operators. In a Lipschitz polyhedron $\Omega$, one can build finite element interpolation, or quasi-interpolation, operators that act on piecewise smooth fields, with range in $\boldsymbol{V}_{h}$. For a polyhedral partition $\mathcal{P}:=\left\{\Omega_{j}\right\}_{j=1, \cdots, J}$, the family of meshes $\left(\mathcal{T}_{h}\right)_{h}$ is said to be conforming if, for all $h$, for all $K \in \mathcal{T}_{h}$, there exists $j_{0}$ such that $K \subset \overline{\Omega_{j_{0}}}$. Let us recall briefly the theory of finite element interpolation. Classically, those results are obtained by studying the properties of the mappings to the reference element, using Sobolev-Slobodeckij seminorms. It holds, for conforming meshes,

$$
\begin{aligned}
& \forall s \in(0,1], \exists \underline{C}_{(\varsigma, s)}^{\text {interp }}>0, \forall \boldsymbol{v} \in \boldsymbol{P} \boldsymbol{H}^{s}(\mathbf{c u r l} ; \Omega), \forall h, \\
& \left\|\boldsymbol{v}-\Pi_{h}^{\text {interp }} \boldsymbol{v}\right\|_{\boldsymbol{H}(\mathbf{c u r l} ; \Omega)} \leq \underline{C}_{(\varsigma, s)}^{\text {interp }} h^{s}\left\{\|\boldsymbol{v}\|_{\underline{\boldsymbol{P H}}^{s}(\Omega)}+\|\operatorname{curl} \boldsymbol{v}\|_{\underline{\boldsymbol{P H}}^{s}(\Omega)}\right\} .
\end{aligned}
$$

In (3.4), the interpolation operator $\Pi_{h}^{\text {interp }}$ is defined in $[29, \S 5.5]$ for $s>\frac{1}{2}$, respectively is the so-called combined interpolation operator of $[12, \S 4.2]$ for $s \leq \frac{1}{2}$. Regarding the theory of finite element quasi-interpolation, a similar result can be derived. Namely, that (3.4) holds, where $\Pi_{h}^{\text {interp }}$ now stands for the quasi-interpolation operator defined in $[20, \S 3.5]$. The two finite element interpolation and quasi-interpolation bounds are identical, bearing in mind that $\Pi_{h}^{\text {interp }}$ is either the interpolation, or the quasi-interpolation, operator. In addition, we note that $\underline{C}_{(\varsigma, s)}^{\text {interp }}$ is not proven to be independent of $s$ in the above mentioned papers. On the other hand, one can check that $\underline{C}_{(\varsigma, s)}^{\text {interp }}$ depends continuously on $s$ in $(0,1)$ with the help of the tools proposed in those papers. For the derivation of those continuous dependence results, we refer precisely to: the proof of Theorem 3.3 in [20] using abstract estimates from [19, §5] for the quasi-interpolation; resp. [12, §4.2] using estimates for the Scott-Zhang interpolation, for the combined interpolation. Both proofs rely on $[9, \S 14.3]$.

With the help of the results on the equivalence of norms of $\S 3.1(1)$, we conclude that one can write (3.4) with the real interpolation norms

$$
\begin{aligned}
& \forall s \in(0,1], \exists C_{(\varsigma, s)}^{\text {interp }}>0, \forall \boldsymbol{v} \in \boldsymbol{P} \boldsymbol{H}^{s}(\mathbf{c u r l} ; \Omega), \forall h, \\
& \left\|\boldsymbol{v}-\Pi_{h}^{\text {interp }} \boldsymbol{v}\right\|_{\boldsymbol{H}(\mathbf{c u r l} ; \Omega)} \leq C_{(\varsigma, s)}^{\text {interp }} h^{s}\|\boldsymbol{v}\|_{\boldsymbol{P} \boldsymbol{H}^{s}(\mathbf{c u r l} ; \Omega)} .
\end{aligned}
$$

Furthermore, one can choose $s \mapsto C_{(\varsigma, s)}^{\text {interp }}$ that is continuous on $\left[s_{0}, s_{1}\right]$, for all $0<$ $s_{0} \leq s_{1}<1$. 
3.4. Extra-regularity of the electric field and convergence rate. Since $\boldsymbol{j}_{\text {ext }} \in \boldsymbol{H}(\operatorname{div} ; \Omega)$, one may prove that the electric field that solves (2.9)-(2.10) enjoys extra smoothness. More precisely, the aim is to prove that

$$
\begin{aligned}
& \exists \tau_{(\varepsilon, \mu, \sigma)}>0, \forall t \in\left(0, \tau_{(\varepsilon, \mu, \sigma)}\right), \exists C_{(\varepsilon, \mu, \sigma, t)}^{\star}, \forall \boldsymbol{j}_{e x t} \in \boldsymbol{H}(\operatorname{div} ; \Omega), \\
& \boldsymbol{e} \in \boldsymbol{P} \boldsymbol{H}^{t}(\mathbf{c u r l} ; \Omega) \text { and }\|\boldsymbol{e}\|_{\boldsymbol{P H}^{t}(\mathbf{c u r l} ; \Omega)} \leq C_{(\varepsilon, \mu, \sigma, t)}^{\star}\left\|\boldsymbol{j}_{\text {ext }}\right\|_{\boldsymbol{H}(\operatorname{div} ; \Omega)} .
\end{aligned}
$$

Above, $\tau_{(\varepsilon, \mu, \sigma)}$ plays the role of a regularity exponent, while $C_{(\varepsilon, \mu, \sigma, s)}^{\star}$ can be seen as a stability constant.

Let $\Theta$ be a set of coefficients $(\varepsilon, \mu, \sigma)$ whose elements are all piecewise smooth on the same partition, and assume that $\underline{\tau}=\inf _{(\varepsilon, \mu, \sigma) \in \Theta} \tau_{(\varepsilon, \mu, \sigma)}>0$, where $\tau_{(\varepsilon, \mu, \sigma)}$ is defined in (3.6), and let $\boldsymbol{j}_{\text {ext }}$, the data, be given. Regrouping all the previous results, one concludes first that for all $(\varepsilon, \mu, \sigma) \in \Theta$, the solution $\boldsymbol{e}$ to (2.9)-(2.10) is in $\bigcap_{s \in[0, \underline{\tau})} \boldsymbol{P} \boldsymbol{H}^{s}(\mathbf{c u r l} ; \Omega)$; and second that one has the error estimates

$$
\begin{aligned}
& \forall s \in(0, \underline{\tau}), \\
& \left\|\boldsymbol{e}-\boldsymbol{e}_{h}\right\|_{\boldsymbol{H}(\mathbf{c u r l} ; \Omega)} \leq C_{(\varepsilon, \mu, \sigma)}^{\sharp} C_{(\varsigma, s)}^{\text {interp }} C_{(\varepsilon, \mu, \sigma, s)}^{\star} h^{s}\left\|\boldsymbol{j}_{\text {ext }}\right\|_{\boldsymbol{H}(\mathrm{div} ; \Omega)} .
\end{aligned}
$$

In the error estimates (3.7), only $C_{(\varepsilon, \mu, \sigma)}^{\sharp}$ and $C_{(\varepsilon, \mu, \sigma, s)}^{\star}$ depend on the coefficients $(\varepsilon, \mu, \sigma)$. Also, for a given $\epsilon \in(0, \underline{\tau})$, since $s \mapsto C_{(\varsigma, s)}^{\text {interp }}$ is continuous for $s>0$, one may replace $C_{(\varsigma, s)}^{\text {interp }}$ by the $s$-independent $\max _{s \in[\epsilon, \underline{\tau})} C_{(\varsigma, s)}^{\text {interp }}$, for all $s \in[\epsilon, \underline{\tau})$.

Our purpose is now to estimate more precisely the constants that appear in (3.3), (3.5), (3.6) and (3.7). The dependency of $C_{(\varepsilon, \mu, \sigma)}^{\sharp}$ on $(\varepsilon, \mu, \sigma)$ is addressed in section 4. For $\tau_{(\varepsilon, \mu, \sigma)}$ and $C_{(\varepsilon, \mu, \sigma, s)}^{\star}$, this dependency can be studied via the global approach, which relies on a decomposition of the electric field, and of its curl, into a regular part, and a gradient part. To obtain this splitting, we adapt [4, Chapter $6]$ to the case of complex-valued coefficients, and in the process we generalize the results of [15] to the case of a non-topologically trivial domain: this is the subject of section 5 . In section 6 , one studies the regularity of the gradient part, where the scalar potential is governed by a second order elliptic PDEs complemented either with Dirichlet boundary conditions (for the electric field) or with Neumann boundary conditions (for its curl). The global approach, in the spirit of $[27,6]$, uses a perturbation argument, where the regularity of the gradient part, ie. of its scalar potential, is derived in comparison to the regularity of the solution to the Laplace equation with the same boundary condition. Indeed, in a domain $\Omega$ and for $L^{2}(\Omega)$ volume data, it is known from [26] that the gradient of the solution to the Laplace equation belongs to $\boldsymbol{H}^{\frac{1}{2}}(\Omega)$. Using interpolation theory, one can find a regularity exponent $\tau_{(\varepsilon, \mu, \sigma)}$ in (3.6) for our problem. Up to our knowledge, this analysis has only been carried out for PDEs with real-valued coefficients. Here, we check in particular that the analysis proposed in [6] can be extended to the case of complex-valued coefficients. The main results are: the derivation of a regularity exponent $\tau_{(\varepsilon, \mu, \sigma)}$ that depends polynomially on the coefficients, and the computation of an upper bound for the stability constant $C_{(\varepsilon, \mu, \sigma, s)}^{\star}$ when $s$ spans $\left(0, \tau_{(\varepsilon, \mu, \sigma)}\right)$. Finally, in section 7 we illustrate the theory on two examples for which the singular behavior can be determined explicitly.

Remark 3.2. For piecewise constant coefficients, there exists an alternative, which focuses on the singular behavior of the gradient part of the solution by finding directly the "best" regularity exponent $\tau_{(\varepsilon, \mu, \sigma)}^{o p t}$ attached to this part of the solution. We call it the local approach, see Appendix B. On the other hand, providing an upper bound with the local approach for $C_{(\varepsilon, \mu, \sigma, s)}^{\star}$ when $s$ spans $\left(0, \tau_{(\varepsilon, \mu, \sigma)}^{o p t}\right)$, is an open question. 
4. Estimating the constant $C_{(\varepsilon, \mu, \sigma)}^{\sharp}$. Let $\boldsymbol{V}:=\boldsymbol{H}_{0}(\mathbf{c u r l} ; \Omega)$ be endowed with $\|\boldsymbol{v}\|_{\boldsymbol{V}}:=\left(\|\boldsymbol{v}\|_{0, \Omega}^{2}+\|\operatorname{curl} \boldsymbol{v}\|_{0, \Omega}^{2}\right)^{1 / 2}$, and $a(\cdot, \cdot)$ be the sesquilinear form on $\boldsymbol{V}$ defined by:

$$
(\boldsymbol{v}, \boldsymbol{w}) \mapsto\left(\mu^{-1} \operatorname{curl} \boldsymbol{v} \mid \operatorname{curl} \boldsymbol{w}\right)_{0, \Omega}-\omega^{2}\left(\varepsilon_{\sigma} \boldsymbol{v} \mid \boldsymbol{w}\right)_{0, \Omega} .
$$

Then, let $C_{(\varepsilon, \mu, \sigma)}^{c o n t}$ be the best continuity constant, or continuity modulus, of $a(\cdot, \cdot)$ :

$$
C_{(\varepsilon, \mu, \sigma)}^{\text {cont }}=\sup _{\boldsymbol{v}, \boldsymbol{w} \in \boldsymbol{V} \backslash\{0\}} \frac{|a(\boldsymbol{v}, \boldsymbol{w})|}{\|\boldsymbol{v}\|_{\boldsymbol{V}}\|\boldsymbol{w}\|_{\boldsymbol{V}}},
$$

resp. $C_{(\varepsilon, \mu, \sigma)}^{c o e r}$ be the best coercivity constant of $a(\cdot, \cdot)$ :

$$
C_{(\varepsilon, \mu, \sigma)}^{c o e r}=\inf _{\boldsymbol{v} \in \boldsymbol{V} \backslash\{0\}} \frac{|a(\boldsymbol{v}, \boldsymbol{v})|}{\|\boldsymbol{v}\|_{\boldsymbol{V}}^{2}} .
$$

Proposition 4.1. Let the coefficients $\varepsilon, \mu$ and $\sigma$ be as in section 2.1. Then the sesquilinear form $a(\cdot, \cdot)$ is continuous, with $C_{(\varepsilon, \mu, \sigma)}^{\text {cont }} \leq \max \left(\omega\left(\omega^{2} \varepsilon_{\text {max }}^{2}+\sigma_{\text {max }}^{2}\right)^{1 / 2}, \mu^{-1}\right)$, and it is coercive with $C_{(\varepsilon, \mu, \sigma)}^{\text {coer }} \geq \frac{1}{2} \sigma_{\min } \min \left(\omega \varepsilon_{\min } \varepsilon_{\max }^{-1}, \mu^{-1}\left(\omega^{2} \varepsilon_{\max }^{2}+\frac{1}{2} \sigma_{\min }^{2}\right)^{-1 / 2}\right)$.

Proof. (We omit the subscript $0, \Omega$ for the $L^{2}(\Omega)$-scalar product and norm). Regarding continuity, given $\boldsymbol{v}, \boldsymbol{w} \in \boldsymbol{V}$, one finds

$$
\begin{aligned}
|a(\boldsymbol{v}, \boldsymbol{w})| & \leq \omega^{2}\left\|\varepsilon_{\sigma}\right\|_{L^{\infty}(\Omega)}\|\boldsymbol{v}\|\|\boldsymbol{w}\|+\mu^{-1}\|\operatorname{curl} \boldsymbol{v}\|\|\operatorname{curl} \boldsymbol{w}\| \\
& \leq \max \left(\omega^{2}\left\|\varepsilon_{\sigma}\right\|_{L^{\infty}(\Omega)}, \mu^{-1}\right)(\|\boldsymbol{v}\|\|\boldsymbol{w}\|+\|\operatorname{curl} \boldsymbol{v}\|\|\operatorname{curl} \boldsymbol{w}\|) \\
& \leq \max \left(\omega^{2}\left\|\varepsilon_{\sigma}\right\|_{L^{\infty}(\Omega)}, \mu^{-1}\right)\|\boldsymbol{v}\|_{\boldsymbol{V}}\|\boldsymbol{w}\|_{\boldsymbol{V}} \\
& \leq \max \left(\omega\left(\omega^{2} \varepsilon_{\text {max }}^{2}+\sigma_{\text {max }}^{2}\right)^{1 / 2}, \mu^{-1}\right)\|\boldsymbol{v}\|_{\boldsymbol{V}}\|\boldsymbol{w}\|_{\boldsymbol{V}}
\end{aligned}
$$

because $\left\|\varepsilon_{\sigma}\right\|_{L^{\infty}(\Omega)} \leq\left(\varepsilon_{\max }^{2}+\omega^{-2} \sigma_{\max }^{2}\right)^{1 / 2}$.

Regarding coercivity, given $\boldsymbol{v} \in \boldsymbol{V}$, il we let $\boldsymbol{c}=\operatorname{curl} \boldsymbol{v}$, one finds

$$
\begin{aligned}
|a(\boldsymbol{v}, \boldsymbol{v})|^{2} & =\left(-\omega^{2}(\varepsilon \boldsymbol{v} \mid \boldsymbol{v})+\mu^{-1}\|\boldsymbol{c}\|^{2}\right)^{2}+\omega^{2}(\sigma \boldsymbol{v} \mid \boldsymbol{v})^{2} \\
& =\omega^{4}(\varepsilon \boldsymbol{v} \mid \boldsymbol{v})^{2}+\mu^{-2}\|\boldsymbol{c}\|^{4}-2 \omega^{2} \mu^{-1}(\varepsilon \boldsymbol{v} \mid \boldsymbol{v})\|\boldsymbol{c}\|^{2}+\omega^{2}(\sigma \boldsymbol{v} \mid \boldsymbol{v})^{2} \\
& \geq\left(\omega^{4}-\omega^{2} \eta\right)(\varepsilon \boldsymbol{v} \mid \boldsymbol{v})^{2}+\mu^{-2}\left(1-\omega^{2} \eta^{-1}\right)\|\boldsymbol{c}\|^{4}+\omega^{2}(\sigma \boldsymbol{v} \mid \boldsymbol{v})^{2},
\end{aligned}
$$

for all $\eta>0$ (Young's inequality). Then,

$$
\begin{aligned}
|a(\boldsymbol{v}, \boldsymbol{v})|^{2} & \geq\left(\omega^{4}-\omega^{2} \eta\right)(\varepsilon \boldsymbol{v} \mid \boldsymbol{v})^{2}+\mu^{-2}\left(1-\omega^{2} \eta^{-1}\right)\|\boldsymbol{c}\|^{4}+\omega^{2} \sigma_{\min }^{2}\|\boldsymbol{v}\|^{4} \\
& \geq \omega^{2} \varepsilon_{\min }^{2}\left(\omega^{2}+\sigma_{\min }^{2} \varepsilon_{\max }^{-2}-\eta\right)\|\boldsymbol{v}\|^{4}+\mu^{-2}\left(1-\omega^{2} \eta^{-1}\right)\|\boldsymbol{c}\|^{4} .
\end{aligned}
$$

As a consequence, choosing $\eta \in\left(\omega^{2}, \omega^{2}+\sigma_{\min }^{2} \varepsilon_{\max }^{-2}\right)$, one derives coercivity. For instance, let $\eta=\omega^{2}+\frac{1}{2} \sigma_{\min }^{2} \varepsilon_{\max }^{-2}$. It follows that

$$
\begin{aligned}
|a(\boldsymbol{v}, \boldsymbol{v})|^{2} & \geq \frac{1}{2} \omega^{2} \sigma_{\min }^{2} \frac{\varepsilon_{\min }^{2}}{\varepsilon_{\max }^{2}}\|\boldsymbol{v}\|^{4}+\frac{\sigma_{\text {min }}^{2}}{2 \mu^{2}\left(\omega^{2} \varepsilon_{\text {max }}^{2}+\frac{1}{2} \sigma_{\text {min }}^{2}\right)}\|\boldsymbol{c}\|^{4} \\
& \geq \frac{\sigma_{\text {min }}^{2}}{2} \min \left(\omega^{2} \frac{\varepsilon_{\text {min }}^{2}}{\varepsilon_{\text {max }}^{2}}, \frac{1}{\mu^{2}\left(\omega^{2} \varepsilon_{\text {max }}^{2}+\frac{1}{2} \sigma_{\text {min }}^{2}\right)}\right)\left(\|\boldsymbol{v}\|^{4}+\|\boldsymbol{c}\|^{4}\right) \\
& \geq \frac{\sigma_{\text {min }}^{2}}{4} \min \left(\omega^{2} \frac{\varepsilon_{\text {min }}^{2}}{\varepsilon_{\text {max }}^{2}}, \frac{1}{\mu^{2}\left(\omega^{2} \varepsilon_{\text {max }}^{2}+\frac{1}{2} \sigma_{\text {min }}^{2}\right)}\right)\|\boldsymbol{v}\|_{\boldsymbol{V}}^{4} .
\end{aligned}
$$

Hence, $|a(\boldsymbol{v}, \boldsymbol{v})| \geq \frac{1}{2} \sigma_{\min } \min \left(\omega \varepsilon_{\min } \varepsilon_{\max }^{-1}, \mu^{-1}\left(\omega^{2} \varepsilon_{\max }^{2}+\frac{1}{2} \sigma_{\min }^{2}\right)^{-1 / 2}\right)\|\boldsymbol{v}\|_{\boldsymbol{V}}^{2}$. 
Corollary 4.2. Let the coefficients $\varepsilon, \mu$ and $\sigma$ be as in section 2.1. Then the error estimate (3.3) holds with

$$
C_{(\varepsilon, \mu, \sigma)}^{\sharp} \leq \frac{2 \max \left(\omega\left(\omega^{2} \varepsilon_{\max }^{2}+\sigma_{\max }^{2}\right)^{1 / 2}, \mu^{-1}\right)}{\sigma_{\min } \min \left(\omega \varepsilon_{\min } \varepsilon_{\max }^{-1}, \mu^{-1}\left(\omega^{2} \varepsilon_{\max }^{2}+\frac{1}{2} \sigma_{\min }^{2}\right)^{-1 / 2}\right)} .
$$

Proof. This is an obvious consequence of the fact that one can choose

$$
C_{(\varepsilon, \mu, \sigma)}^{\sharp}=\frac{C_{(\varepsilon, \mu, \sigma)}^{c o n t}}{C_{(\varepsilon, \mu, \sigma)}^{c o e r}}
$$

in the error estimate (3.3).

5. Splitting into a regular part and a gradient part. Below, we recall some results of [4], and we adapt them to the case of complex-valued coefficients if necessary. Let $\xi$ be a coefficient defined on $\Omega$, we assume in the current section that $\xi$ fulfills:

$$
\left\{\begin{array}{l}
\xi \text { is a complex-valued measurable scalar field on } \Omega, \xi, \xi^{-1} \in L^{\infty}(\Omega), \\
\exists \xi_{-}>0, \theta^{\star} \in[0,2 \pi), \Re\left(\exp \left(-\imath \theta^{\star}\right) \xi\right) \geq \xi_{-} \text {a.e. in } \Omega .
\end{array}\right.
$$

LEMma 5.1. Let the coefficients $\varepsilon$ and $\sigma$ be as in section 2.1. Then $\xi=\varepsilon_{\sigma}$ fulfills (5.1), where $\theta^{\star}$ can be any element of $[0, \pi / 2]$.

Remark 5.2. In other words, $\xi=\varepsilon_{\sigma}$ belongs to a subclass of those coefficients that are defined by (5.1). In the case where $\sigma \geq 0$ (in particular, in the non-conducting case, that is when it holds that $\sigma=0$ on some region of $\Omega$ ), the above result still holds for all $\theta^{\star} \in[0, \pi / 2)$. On the other hand, a real-valued, sign-changing coefficient $\xi$ does not fulfill $(5.1)$. We refer to $[8,7,18,10]$ for those more "exotic" configurations of Maxwell's equations, in which $\varepsilon$ and/or $\mu$ are real-valued and exhibit a sign-change.

Proof. One has $\varepsilon_{\sigma}, \varepsilon_{\sigma}^{-1} \in L^{\infty}(\Omega)$. The result follows from $\Re\left(\exp \left(-\imath \theta^{\star}\right) \varepsilon_{\sigma}\right) \geq$ $\cos \theta^{\star} \varepsilon_{\min }+\sin \theta^{\star} \sigma_{\min } / \omega>0$ a.e. in $\Omega$.

Define

$$
\begin{aligned}
& \boldsymbol{X}_{D i r}(\Omega, \xi):=\left\{\boldsymbol{v} \in \boldsymbol{H}_{0}(\operatorname{curl} ; \Omega): \xi \boldsymbol{v} \in \boldsymbol{H}(\operatorname{div} ; \Omega)\right\} \\
& \boldsymbol{X}_{N e u}(\Omega, \xi):=\left\{\boldsymbol{v} \in \boldsymbol{H}(\operatorname{curl} ; \Omega): \xi \boldsymbol{v} \in \boldsymbol{H}_{0}(\operatorname{div} ; \Omega)\right\}
\end{aligned}
$$

The function spaces $\boldsymbol{X}_{D i r}(\Omega, \xi)$ and $\boldsymbol{X}_{N e u}(\Omega, \xi)$ are endowed with the graph norm $\boldsymbol{v} \mapsto\left(\|\boldsymbol{v}\|_{\boldsymbol{H}(\mathbf{c u r l} ; \Omega)}^{2}+\|\xi \boldsymbol{v}\|_{\boldsymbol{H}(\mathrm{div} ; \Omega)}^{2}\right)^{1 / 2}$. In the particular case where $\xi$ is equal to 1 , one writes $\boldsymbol{X}_{B}(\Omega)$ instead of $\boldsymbol{X}_{B}(\Omega, 1)$, for $B \in\{D i r, N e u\}$. We also define the subspaces of regular fields, resp. the null subspaces:

$$
\begin{aligned}
& \boldsymbol{H}_{B}(\Omega):=\boldsymbol{X}_{B}(\Omega) \cap \boldsymbol{H}^{1}(\Omega), B \in\{D i r, N e u\} \\
& \boldsymbol{Z}_{B}(\Omega):=\left\{\boldsymbol{v} \in \boldsymbol{X}_{B}(\Omega): \operatorname{curl} \boldsymbol{v}=0, \operatorname{div} \boldsymbol{v}=0 \text { in } \Omega\right\}, B \in\{D i r, N e u\}
\end{aligned}
$$

In our case, both $\varepsilon_{\sigma}$ and $\mu$ fulfill (5.1) and moreover, since $\boldsymbol{j}_{\text {ext }} \in \boldsymbol{H}$ (div; $\Omega$ ), we note that the solution $\boldsymbol{e}$ to (2.9) is such that $\boldsymbol{e} \in \boldsymbol{X}_{\text {Dir }}\left(\Omega, \varepsilon_{\sigma}\right)$, and $\mu^{-1} \operatorname{curl} \boldsymbol{e} \in$ $\boldsymbol{X}_{\text {Neu }}(\Omega, \mu)$. 
5.1. Geometric framework. The domain $\Omega$ can be topologically non-trivial, or with a non-connected boundary. Regarding the first item, we assume that:

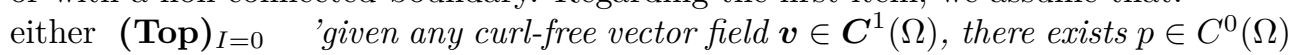
such that $\boldsymbol{v}=\nabla p$ in $\Omega$ ';

or (Top) $)_{I>0}$ 'there exist I non-intersecting manifolds, $\Sigma_{1}, \ldots, \Sigma_{I}$, with boundaries $\partial \Sigma_{i} \subset \partial \Omega$, such that, if we let $\dot{\Omega}=\Omega \backslash \bigcup_{i=1}^{I} \Sigma_{i}$, given any curl-free vector field $\boldsymbol{v}$, there exists $\dot{p} \in C^{0}(\dot{\Omega})$ such that $\boldsymbol{v}=\nabla \dot{p}$ in $\dot{\Omega}$ '.

When $I=0, \dot{\Omega}=\Omega$. For short, we write (Top) $)_{I}$ to cover both instances. One can build cuts that are piecewise plane, see [23, Chapter 6$]$. Finally, we assume that $\dot{\Omega}$ is a connected set. For the polyhedral model problem, we assume that (Top) $)_{I}$ is fulfilled. The domain $\Omega$ is said to be topologically trivial when $I=0$. When $I>0$, the set $\dot{\Omega}$ has pseudo-Lipschitz boundary in the sense of [3].

The a priori regularity of elements of $\boldsymbol{X}_{\text {Dir }}(\Omega)$ and $\boldsymbol{X}_{\text {Neu }}(\Omega)$ is described in [3, Remark 2.16 and Proposition 3.7]. Below, $\subset$ refers to an algebraical and topological embedding.

Proposition 5.3. Let $\Omega$ be a Lipschitz polyhedron: there exists $\sigma_{\text {Dir }} \in\left(\frac{1}{2}, 1\right]$ such that it holds that $\boldsymbol{X}_{\text {Dir }}(\Omega) \subset \boldsymbol{H}^{\sigma_{\text {Dir }}}(\Omega)$. Assume in addition that $(\mathbf{T o p})_{I}$ is fulfilled: there exists $\sigma_{\mathrm{Neu}} \in\left(\frac{1}{2}, 1\right]$ such that it holds that $\boldsymbol{X}_{\mathrm{Neu}}(\Omega) \subset \boldsymbol{H}^{\sigma_{\mathrm{Neu}}}(\Omega)$.

Let $\Omega$ be a domain: the embeddings hold with $\sigma_{D i r}=\sigma_{N e u}=\frac{1}{2}$.

Corollary 5.4. With the same assumptions as in Proposition 5.3, it holds that $\boldsymbol{Z}_{\text {Dir }}(\Omega) \subset \boldsymbol{H}^{\sigma_{\text {Dir }}}(\Omega)$ and $\boldsymbol{Z}_{N e u}(\Omega) \subset \boldsymbol{H}^{\sigma_{N e u}}(\Omega)$.

Finally, one can prove that the null spaces $\boldsymbol{Z}_{D i r}(\Omega)$ and $\boldsymbol{Z}_{N e u}(\Omega)$ are finite dimensional vector spaces.

5.2. Splittings of fields. We provide now splittings into a regular part, and a gradient part, of elements of $\boldsymbol{X}_{\text {Dir }}(\Omega, \xi)$ ("electric case"), resp. of elements of $\boldsymbol{X}_{N e u}(\Omega, \xi)$ ("magnetic case"), called regular/gradient splittings. The proofs can be found in $\S 6.1 .6$ and $\S 6.2 .6$ of [4]. We provide some comments on these splittings below.

THEOREM 5.5. Let $\Omega$ be a domain such that (Top) ${ }_{I}$ is fulfilled, and assume that $\xi$ fulfills (5.1). Then, there exists a continuous splitting operator acting from $\boldsymbol{X}_{D i r}(\Omega, \xi)$ to $\boldsymbol{H}_{\text {Dir }}(\Omega) \times \boldsymbol{Z}_{\text {Dir }}(\Omega) \times H_{0}^{1}(\Omega)$.

More precisely, given $\boldsymbol{v} \in \boldsymbol{X}_{\text {Dir }}(\Omega, \xi)$,

$$
\exists\left(\boldsymbol{v}_{r e g}, \boldsymbol{z}, p_{0}\right) \in \boldsymbol{H}_{D i r}(\Omega) \times \boldsymbol{Z}_{D i r}(\Omega) \times H_{0}^{1}(\Omega), \boldsymbol{v}=\boldsymbol{v}_{r e g}+\boldsymbol{z}+\nabla p_{0} \text { in } \Omega .
$$

One has

$$
\left\|\boldsymbol{v}_{r e g}\right\|_{1, \Omega}+\left\|\boldsymbol{v}_{r e g}\right\|_{\boldsymbol{X}_{\text {Dir }}(\Omega)}+\|\boldsymbol{z}\|_{\sigma_{D i r}, \Omega}+\left\|\boldsymbol{v}_{r e g}+\boldsymbol{z}\right\|_{1 / 2, \Omega} \leq C_{\boldsymbol{X}}^{D i r}\|\boldsymbol{v}\|_{\boldsymbol{H}(\mathbf{c u r l} ; \Omega)} .
$$

The scalar field $p_{0}$ is governed by the variational formulation:

$$
\left\{\begin{array}{l}
\text { Find } p_{0} \in H_{0}^{1}(\Omega) \text { such that } \\
\quad\left(\xi \nabla p_{0} \mid \nabla \psi\right)_{0, \Omega}=-(\xi \boldsymbol{z} \mid \nabla \psi)_{0, \Omega}-\left(\xi \boldsymbol{v}_{r e g} \mid \nabla \psi\right)_{0, \Omega} \\
\quad-(\operatorname{div} \xi \boldsymbol{v} \mid \psi)_{0, \Omega}, \quad \forall \psi \in H_{0}^{1}(\Omega) .
\end{array}\right.
$$

THEOREm 5.6. Let $\Omega$ be a domain such that (Top) $)_{I}$ is fulfilled, and assume that $\xi$ fulfills (5.1). Then, there exists a continuous splitting operator acting from $\boldsymbol{X}_{N e u}(\Omega, \xi)$ to $\boldsymbol{H}_{z m v}^{1}(\Omega) \times \boldsymbol{Z}_{N e u}(\Omega) \times H_{z m v}^{1}(\Omega)$.

More precisely, given $\boldsymbol{v} \in \boldsymbol{X}_{N e u}(\Omega, \xi)$,

(5.5) $\exists\left(\boldsymbol{w}_{r e g}, \boldsymbol{z}, q_{0}\right) \in \boldsymbol{H}_{z m v}^{1}(\Omega) \times \boldsymbol{Z}_{N e u}(\Omega) \times H_{z m v}^{1}(\Omega), \boldsymbol{v}=\boldsymbol{w}_{r e g}+\boldsymbol{z}+\nabla q_{0}$ in $\Omega$. 
One has

$$
\left\|\boldsymbol{w}_{r e g}\right\|_{1, \Omega}+\left\|\boldsymbol{w}_{r e g}\right\|_{\boldsymbol{X}_{N e u}(\Omega)}+\|\boldsymbol{z}\|_{\sigma_{N e u}, \Omega}+\left\|\boldsymbol{w}_{r e g}+\boldsymbol{z}\right\|_{1 / 2, \Omega} \leq C_{\boldsymbol{X}}^{N e u}\|\boldsymbol{v}\|_{\boldsymbol{H}(\mathbf{c u r l} ; \Omega)} .
$$

The scalar field $q_{0}$ is governed by the variational formulation:

$$
\left\{\begin{array}{l}
\text { Find } q_{0} \in H_{z m v}^{1}(\Omega) \text { such that } \\
\left(\xi \nabla q_{0} \mid \nabla \psi\right)_{0, \Omega}=-(\xi \boldsymbol{z} \mid \nabla \psi)_{0, \Omega}-\left(\xi \boldsymbol{w}_{r e g} \mid \nabla \psi\right)_{0, \Omega} \\
\quad-(\operatorname{div} \xi \boldsymbol{v} \mid \psi)_{0, \Omega}, \quad \forall \psi \in H_{z m v}^{1}(\Omega) .
\end{array}\right.
$$

In the splitting (5.2) of $\boldsymbol{v} \in \boldsymbol{X}_{\text {Dir }}(\Omega, \xi)$, all three terms $\boldsymbol{v}_{r e g}, \boldsymbol{z}, \nabla p_{0}$ have vanishing tangential components on the boundary $\partial \Omega$, whereas in the splitting (5.5) of $\boldsymbol{v} \in$ $\boldsymbol{X}_{N e u}(\Omega, \xi), \boldsymbol{w}_{r e g}$ does not verify a homogeneous boundary condition in general. Since $\xi$ fulfills (5.1), both variational formulations (5.4) and (5.7) are well-posed. Finally, we note that regarding the a priori regularity in (5.2), one has $\boldsymbol{v}_{r e g} \in \boldsymbol{H}^{1}(\Omega)$ and $\boldsymbol{z} \in$ $\boldsymbol{H}^{\sigma_{\operatorname{Dir}}}(\Omega)$. Likewise, regarding the a priori regularity in (5.5), one has $\boldsymbol{w}_{\text {reg }} \in \boldsymbol{H}^{1}(\Omega)$ and $\boldsymbol{z} \in \boldsymbol{H}^{\sigma_{\mathrm{Neu}}}(\Omega)$.

5.3. Comments. One may easily generalize the splitting theory to the case where $\xi$ is a complex-valued, measurable, tensor field. As a matter of fact, it is straightforward to check that if $\xi$ fulfills:

$$
\left\{\begin{array}{l}
\xi \text { is a complex-valued measurable tensor field on } \Omega, \xi, \xi^{-1} \in \mathbb{L}^{\infty}(\Omega), \\
\exists \xi_{-}>0, \theta^{\star} \in[0,2 \pi), \forall \boldsymbol{z} \in \mathbb{C}^{3}, \Re\left(\exp \left(-\imath \theta^{\star}\right) \xi \boldsymbol{z} \cdot \overline{\boldsymbol{z}}\right) \geq \xi_{-}|\boldsymbol{z}|^{2} \text { a.e. in } \Omega
\end{array}\right.
$$

then the conclusions of Theorems 5.5 and 5.6 still apply. Obviously, (5.4) and (5.7) are well-posed.

In the special case where $\xi$ is a normal tensor field $\left(\xi^{*} \xi=\xi \xi^{*}\right.$ a.e. in $\left.\Omega\right)$, or equivalently there exists a unitary tensor field $\mathbb{U}$ and a diagonal tensor field $\mathbb{D}$ such that $\xi=\mathbb{U}^{-1} \mathbb{D U}$ a.e. in $\Omega$, one can reformulate the second line of the above condition as

$$
\exists \xi_{-}>0, \theta^{\star} \in[0,2 \pi), \min _{k=1,2,3} \Re\left(\exp \left(-\imath \theta^{\star}\right) \mathbb{D}_{k k}\right) \geq \xi_{-} \text {a.e. in } \Omega .
$$

6. The global approach for finding a regularity exponent $\tau_{(\varepsilon, \mu, \sigma)}$ and a stability constant $C_{(\varepsilon, \mu, \sigma, s)}^{\star}$. To estimate the regularity exponent, we adapt some results of [6] to the case of complex-valued coefficients. Let $\xi$ be a coefficient defined on $\Omega$, we assume in the current section that $\xi$ fulfills (5.1). This assumption prescribes that

$$
\begin{aligned}
& \xi \in\left\{z=\rho \exp (\imath \theta), \rho \in\left[\xi_{-}, \xi_{\text {max }}\right], \theta \in\left[\theta_{\min }, \theta_{\max }\right]\right\} \text { a.e. in } \Omega, \text { where } \\
& \xi_{\text {max }}:=\|\xi\|_{L^{\infty}(\Omega)}, \text { and } 0 \leq \theta_{\max }-\theta_{\min } \leq 2 \arccos \left(\frac{\xi_{-}}{\xi_{\max }}\right) .
\end{aligned}
$$

In other words, since $\arccos \left(\xi_{-} / \xi_{\max }\right)<\pi / 2$ the coefficient $\xi$ takes its values in some open, half plane in $\mathbb{C}$. If the coefficients $\varepsilon$ and $\sigma$ are as in section 2.1, then $\xi=\varepsilon_{\sigma}$ takes its values in some open, quarter plane in $\mathbb{C}$.

We recall that $\boldsymbol{e} \in \boldsymbol{X}_{\text {Dir }}\left(\Omega, \varepsilon_{\sigma}\right)$ and $\mu^{-1} \operatorname{curl} \boldsymbol{e} \in \boldsymbol{X}_{N e u}(\Omega, \mu)$. Hence, according to Theorems 5.5 and 5.6 , we may write:

$$
\begin{array}{r}
\boldsymbol{e}=\boldsymbol{e}_{r e g}+\boldsymbol{z}_{e}+\nabla p_{0} \text { in } \Omega, \quad \boldsymbol{e}_{r e g} \in \boldsymbol{H}^{1}(\Omega), \boldsymbol{z}_{e} \in \boldsymbol{H}^{\sigma_{\text {Dir }}}(\Omega) ; \\
\mu^{-1} \text { curl } \boldsymbol{e}=\boldsymbol{c}_{r e g}+\boldsymbol{z}_{c}+\nabla q_{0} \text { in } \Omega, \boldsymbol{c}_{r e g} \in \boldsymbol{H}^{1}(\Omega), \boldsymbol{z}_{c} \in \boldsymbol{H}^{\sigma_{N e u}}(\Omega) .
\end{array}
$$


In addition, it holds that

$$
\begin{aligned}
& \left\|\boldsymbol{e}_{r e g}\right\|_{1, \Omega}+\left\|\boldsymbol{e}_{r e g}\right\|_{\boldsymbol{X}_{D i r}(\Omega)}+\left\|\boldsymbol{z}_{e}\right\|_{\sigma_{D i r}, \Omega}+\left\|\boldsymbol{e}_{r e g}+\boldsymbol{z}_{e}\right\|_{1 / 2, \Omega} \leq C_{\boldsymbol{X}}^{D i r}\|\boldsymbol{e}\|_{\boldsymbol{H}(\mathbf{c u r l} ; \Omega)}, \\
& \left\|\boldsymbol{c}_{r e g}\right\|_{1, \Omega}+\left\|\boldsymbol{c}_{r e g}\right\|_{\boldsymbol{X}_{\text {Neu }}(\Omega)}+\left\|\boldsymbol{z}_{c}\right\|_{\sigma_{N e u}, \Omega}+\left\|\boldsymbol{c}_{r e g}+\boldsymbol{z}_{c}\right\|_{1 / 2, \Omega} \leq C_{\boldsymbol{X}}^{N e u} \| \mu^{-1} \text { curl } \boldsymbol{e} \|_{\boldsymbol{H}(\mathbf{c u r l} ; \Omega)} .
\end{aligned}
$$

It now remains to evaluate the regularity and norm of the gradient parts $\nabla p_{0}$ and $\nabla q_{0}$. Note that $p_{0}$ is governed by the second-order scalar PDE (5.4) with Dirichlet boundary condition, while $q_{0}$ is governed by the second-order scalar PDE (5.7) with Neumann boundary condition. We will use this vocabulary in the following to address both cases.

6.1. Preliminary results. To start with, given $\mathcal{O} \subset \Omega$ a non-empty connected open subset of $\mathbb{R}^{3}$ with Lipschitz boundary, let $\mathcal{H}^{0}(\mathcal{O})$ be equal to $L^{2}(\mathcal{O})$ in the Dirichlet case, resp. $L_{z m v}^{2}(\mathcal{O})$ in the Neumann case, and $\mathcal{H}^{1}(\mathcal{O})$ be equal to $H_{0}^{1}(\mathcal{O})$ in the Dirichlet case, resp. $H_{z m v}^{1}(\mathcal{O})$ in the Neumann case. We equip $\mathcal{H}^{1}(\mathcal{O})$ with the norm $\|v\|_{\mathcal{H}^{1}(\mathcal{O})}:=\|\nabla v\|_{0, \mathcal{O}}$.

Then, for $s \in(0,1)$, we introduce $\mathcal{H}^{s}(\mathcal{O})$, the Sobolev space obtained by the real interpolation method between $\mathcal{H}^{1}(\mathcal{O})$ and $\mathcal{H}^{0}(\mathcal{O})$ : if needed, we distinguish the two cases by writing $\mathcal{H}_{D i r}^{s}(\mathcal{O})$, resp. $\mathcal{H}_{\text {Neu }}^{s}(\mathcal{O})$. In particular, by definition (cf. §3.1), it holds that $\mathcal{H}_{D i r}^{s}(\mathcal{O})=\widetilde{H}^{s}(\mathcal{O})$ for all $s \in[0,1]$, and we recall that $H^{s}(\mathcal{O})=\widetilde{H}^{s}(\mathcal{O})$ for all $s \in\left[0, \frac{1}{2}\right)$.

We denote by $\mathcal{H}^{-s}(\mathcal{O})$ the dual space of $\mathcal{H}^{s}(\mathcal{O})$, for $s \in[0,1]$. Finally, for $s \in[0,1]$, we define $\mathcal{H}^{1+s}(\mathcal{O}):=\left\{v \in \mathcal{H}^{1}(\mathcal{O})\right.$ s.t. $\left.\nabla v \in \boldsymbol{H}^{s}(\mathcal{O})\right\}$, equipped with the norm $\|v\|_{\mathcal{H}^{1+s}(\mathcal{O})}:=\|\nabla v\|_{s, \mathcal{O}}$.

Lemma 6.1. Given $s \in[0,1]$, there exists $C_{(s)}^{P}>0$ such that

$$
\forall v \in \mathcal{H}^{1+s}(\mathcal{O}), \quad\|v\|_{\mathcal{H}^{1+s}(\mathcal{O})} \leq\|v\|_{1+s, \mathcal{O}} \leq C_{(s)}^{P}\|v\|_{\mathcal{H}^{1+s}(\mathcal{O})} .
$$

Proof. The result is obvious for $s \in\{0,1\}$, according to the Poincaré inequality. We let now $s \in(0,1)$.

For the left inequality, notice that

$$
\forall v \in H^{1}(\mathcal{O}),\|\nabla v\|_{0, \Omega} \leq\|v\|_{1, \Omega} ; \quad \forall v \in H^{2}(\mathcal{O}),\|\nabla v\|_{1, \Omega} \leq\|v\|_{2, \Omega} .
$$

As a consequence, the left inequality follows. This is the so-called exact sequence property. Following Appendix A, if we let $v \in \mathcal{H}^{1+s}(\mathcal{O})$ :

$$
\begin{aligned}
& \|v\|_{\mathcal{H}^{1+s}(\mathcal{O})}:=\|\nabla v\|_{s, \mathcal{O}} \\
& =\left\|t^{-s} \inf _{\substack{\nabla v=\boldsymbol{v}_{0}+\boldsymbol{v}_{1} \\
\boldsymbol{v}_{0} \in \boldsymbol{L}^{2}(\mathcal{O}), \boldsymbol{v}_{1} \in \boldsymbol{H}^{1}(\mathcal{O})}}\left(\left\|\boldsymbol{v}_{0}\right\|_{0, \mathcal{O}}^{2}+t^{2}\left\|\boldsymbol{v}_{1}\right\|_{1, \mathcal{O}}^{2}\right)^{1 / 2}\right\|_{L^{2}\left(0, \infty ; \frac{d t}{t}\right)} \\
& \leq\left\|t^{-s} \inf _{\substack{v=v_{0}+v_{1}\\
}}\left(\left\|\nabla v_{0}\right\|_{0, \mathcal{O}}^{2}+t^{2}\left\|\nabla v_{1}\right\|_{1, \mathcal{O}}^{2}\right)^{1 / 2}\right\|_{L^{2}\left(0, \infty ; \frac{d t}{t}\right)} \\
& v_{0} \in H^{1}(\mathcal{O}), v_{1} \in H^{2}(\mathcal{O}) \\
& \text { (cf. above) } \leq\left\|t^{-s} \inf _{\substack{v=v_{0}+v_{1} \\
v_{0} \in H^{1}(\mathcal{O}), v_{1} \in H^{2}(\mathcal{O})}}\left(\left\|v_{0}\right\|_{1, \mathcal{O}}^{2}+t^{2}\left\|v_{1}\right\|_{2, \mathcal{O}}^{2}\right)^{1 / 2}\right\|_{L^{2}\left(0, \infty ; \frac{d t}{t}\right)} \\
& =:\|v\|_{1+s, \mathcal{O}} \text {. }
\end{aligned}
$$

For the right inequality, let us introduce the Poincaré constant:

$$
\mathcal{C}:=\sup _{\left.v \in \mathcal{H}^{1}(\mathcal{O})\right) \backslash\{0\}} \frac{\|v\|_{0, \mathcal{O}}}{\|\nabla v\|_{0, \mathcal{O}}} .
$$


Using the equivalence of norms, the definition of $\|\cdot\|_{\underline{H}^{1+s}(\mathcal{O})}$, the Poincaré inequality, and finally the equivalence of norms again, we find:

$$
\begin{aligned}
\|v\|_{1+s, \mathcal{O}} & \leq M_{(1+s)}\|v\|_{\underline{H}^{1+s}(\mathcal{O})}:=M_{(1+s)}\left(\|v\|_{1, \mathcal{O}}^{2}+|\nabla v|_{\underline{H}^{s}(\mathcal{O})}^{2}\right)^{1 / 2} \\
& \leq M_{(1+s)}\left(\left(1+\mathcal{C}^{2}\right)\|\nabla v\|_{0, \mathcal{O}}^{2}+|\nabla v|_{\underline{H}^{s}(\mathcal{O})}^{2}\right)^{1 / 2} \\
& \leq M_{(1+s)}\left(1+\mathcal{C}^{2}\right)^{1 / 2}\|\nabla v\|_{\underline{H}^{s}(\mathcal{O})} \\
& \leq M_{(1+s)}\left(1+\mathcal{C}^{2}\right)^{1 / 2} m_{(s)}^{-1}\|\nabla v\|_{s, \mathcal{O}}=: M_{(1+s)}\left(1+\mathcal{C}^{2}\right)^{1 / 2} m_{(s)}^{-1}\|v\|_{\mathcal{H}^{1+s}(\mathcal{O})} .
\end{aligned}
$$

Hence, one may choose $C_{(s)}^{P}=M_{(1+s)}\left(1+\mathcal{C}^{2}\right)^{1 / 2} m_{(s)}^{-1}$.

If we let $\underline{s} \in[0,1]$, we want to find the a priori regularity of the solution to

$$
\left\{\begin{array}{l}
\text { Find } u \in \mathcal{H}^{1}(\Omega) \text { such that } \\
(\xi \nabla u \mid \nabla v)_{0, \Omega}=\langle f, v\rangle_{\mathcal{H}^{1}(\Omega)}, \forall v \in \mathcal{H}^{1}(\Omega)
\end{array}\right.
$$

and $f$ is some data in $\mathcal{H}^{-\underline{s}}(\Omega)$.

If $\xi$ is constant on $\Omega$, that is if one considers the Laplace operator with Dirichlet boundary condition, or with Neumann boundary condition, then one may apply the classical results of [26] or [32] (see [6, p. 504]). See also Proposition 6.7 below. In the statement of the next Theorem, the constant $c^{L a p}(s)$ depends on $\Omega$. For the sake of conciseness, we omit this dependence.

TheOREM 6.2. Let $\xi \neq 0$ be constant on $\Omega$. Then, for all $s \in\left[0, \frac{1}{2}\right)$, there exists $c(s):=c^{\text {Lap }}(s)>0$ such that for all $f \in \mathcal{H}^{s-1}(\Omega)$, the solution $u \in \mathcal{H}^{1}(\Omega)$ to (6.4) belongs to $\mathcal{H}^{s+1}(\Omega)$, and

$$
\|u\|_{\mathcal{H}^{s+1}(\Omega)} \leq \frac{c(s)}{\xi_{\max }}\|f\|_{\mathcal{H}^{s-1}(\Omega)} .
$$

DeFinition 6.3. Let the coefficient $\xi$ fulfill (5.1). We say that $\xi$ fulfills the coefficient assumption if there exists a partition $\mathcal{P}$ of $\Omega$ such that $\xi \in P W^{1, \infty}(\Omega)$.

If $\xi$ fulfills the coefficient assumption on a partition, then $\xi^{-1}$ fulfills the coefficient assumption on the same partition.

From now on in the current section, we consider the case where $\xi \neq 0$ is a scalar, non-constant, complex-valued coefficient that fulfills the coefficient assumption on a partition $\mathcal{P}:=\left\{\Omega_{j}\right\}_{j=1, \cdots, J}$. In [6], the authors study the case of a symmetric-tensor, real-valued coefficient $\xi$. There are similarities between the two cases, and also some differences, that are highlighted below. We refer to $\S 6.5$ for a generalization to the case of a normal-tensor, complex-valued coefficient $\xi$. Let

$$
\Lambda_{\xi}:=\frac{|\xi|_{P W^{1, \infty}(\Omega)}}{\xi_{\max }} .
$$

By definition, it holds that $\|\xi\|_{P W^{1, \infty}(\Omega)}=\xi_{\max }\left(1+\Lambda_{\xi}\right)$. For a piecewise constant coefficient $\xi$, one has $\Lambda_{\xi}=0$. Otherwise, $\Lambda_{\xi}>0$.

For $s \in\left[0, \frac{1}{2}\right)$, choosing $\mathcal{O} \in\left\{\Omega_{j}, 1 \leq j \leq J\right\}$, we denote by $D_{j}^{s}$ the norm of the 
natural embedding of $\boldsymbol{H}^{s}\left(\Omega_{j}\right)$ into $\widetilde{\boldsymbol{H}}^{s}\left(\Omega_{j}\right)$ :

$$
\begin{aligned}
D_{j}^{s} & :=\sup _{\boldsymbol{v}_{j} \in \boldsymbol{H}^{s}\left(\Omega_{j}\right) \backslash\{0\}} \frac{\left\|\boldsymbol{v}_{j}\right\|_{\widetilde{\boldsymbol{H}}^{s}\left(\Omega_{j}\right)}}{\left\|\boldsymbol{v}_{j}\right\|_{s, \Omega_{j}}}, 1 \leq j \leq J ; \\
D_{s} & :=\max \left(1, \max _{1 \leq j \leq J} D_{j}^{s}\right) \geq 1 .
\end{aligned}
$$

Remark 6.4. It holds that $\lim _{s \rightarrow \frac{1}{2}} D_{j}^{s}=+\infty$, because constant, non-vanishing fields defined on $\Omega_{j}$ belong to $\boldsymbol{H}^{\frac{1}{2}}\left(\Omega_{j}\right)$, but not to $\widetilde{\boldsymbol{H}}^{\frac{1}{2}}\left(\Omega_{j}\right)$.

Also, we denote the Poincaré constants by:

$$
C_{j}:=\sup _{\boldsymbol{v}_{j} \in \boldsymbol{H}_{0}^{1}\left(\Omega_{j}\right) \backslash\{0\}} \frac{\left\|\boldsymbol{v}_{j}\right\|_{0, \Omega_{j}}}{\left\|\nabla \boldsymbol{v}_{j}\right\|_{0, \Omega_{j}}}, 1 \leq j \leq J ; C:=\max _{1 \leq j \leq J} C_{j}>0 .
$$

We note that, obviously, the constants $\Lambda_{\xi},\left(D_{j}^{s}\right)_{j}, D_{s},\left(C_{j}\right)_{j}$ and $C$ all depend on $\Omega$, and on the partition $\mathcal{P}$. These dependences are omitted.

Then, we define the multiplicative operator $m_{\xi} \in \mathcal{L}\left(\boldsymbol{L}^{2}(\Omega), \boldsymbol{L}^{2}(\Omega)\right)$ by: $m_{\xi} \boldsymbol{v}(\boldsymbol{x})=$ $\xi(\boldsymbol{x}) \boldsymbol{v}(\boldsymbol{x})$, for all $\boldsymbol{v} \in \boldsymbol{L}^{2}(\Omega)$, a.e. $\boldsymbol{x} \in \Omega$. One may now adapt the proof of Proposition 2.1 of [6] to the complex-valued case, to find...

Proposition 6.5. Let $\xi$ fulfill the coefficient assumption. Then, for all $s \in\left[0, \frac{1}{2}\right)$, it holds that $m_{\xi} \in \mathcal{L}\left(\boldsymbol{H}^{s}(\Omega), \boldsymbol{H}^{s}(\Omega)\right)$ and in addition,

$$
\left\|m_{\xi}\right\|_{\mathcal{L}\left(\boldsymbol{H}^{s}(\Omega), \widetilde{\boldsymbol{H}}^{s}(\Omega)\right)} \leq \xi_{\max } N_{\xi}^{s} \text {, where } N_{\xi}^{s}:=D_{s}\left(2\left(1+C^{2} \Lambda_{\xi}^{2}\right)\right)^{s / 2} .
$$

Furthermore, for all $r \in\left[0, \frac{1}{2}\right)$, it holds that

$$
\left\|m_{\xi}\right\|_{\mathcal{L}\left(\boldsymbol{H}^{s}(\Omega), \widetilde{\boldsymbol{H}}^{s}(\Omega)\right)} \leq \xi_{\max }\left(N_{\xi}^{r}\right)^{s / r}, \forall s \in[0, r] .
$$

We then recall the technical Lemmas 3.1 and 3.2 of [6], which are independent of the coefficient $\xi$. Introduce the operator $D \in \mathcal{L}\left(\boldsymbol{L}^{2}(\Omega), \mathcal{H}^{-1}(\Omega)\right)$ defined by

$$
\langle D \boldsymbol{v}, q\rangle_{\mathcal{H}^{1}(\Omega)}=(\boldsymbol{v} \mid \nabla q)_{0, \Omega}, \forall \boldsymbol{v} \in \boldsymbol{L}^{2}(\Omega), \forall q \in \mathcal{H}^{1}(\Omega) .
$$

Proposition 6.6. For all $s \in[0,1]$, one has

$$
D \in \mathcal{L}\left(\widetilde{\boldsymbol{H}}^{s}(\Omega), \mathcal{H}^{s-1}(\Omega)\right) \text { and }\|D\|_{\mathcal{L}\left(\widetilde{\boldsymbol{H}}^{s}(\Omega), \mathcal{H}^{s-1}(\Omega)\right)} \leq 1 .
$$

Introduce the operator $L \in \mathcal{L}\left(\mathcal{H}^{-1}(\Omega), \mathcal{H}^{1}(\Omega)\right)$ defined by

$$
(\nabla(L v) \mid \nabla q)_{0, \Omega}=\langle v, q\rangle_{\mathcal{H}^{1}(\Omega)}, \forall v \in \mathcal{H}^{-1}(\Omega), \forall q \in \mathcal{H}^{1}(\Omega) .
$$

One may rephrase Theorem 6.2 (with $\xi=1$ ) as follows.

Proposition 6.7. For all $r \in\left[0, \frac{1}{2}\right)$, one has $L \in \mathcal{L}\left(\mathcal{H}^{r-1}(\Omega), \mathcal{H}^{r+1}(\Omega)\right)$ and there exists $K_{r} \geq 1$ such that it holds that:

$$
\begin{aligned}
& \|L\|_{\mathcal{L}\left(\mathcal{H}^{r-1}(\Omega), \mathcal{H}^{r+1}(\Omega)\right)} \leq K_{r} \\
& \text { for all } s \in[0, r],\|L\|_{\mathcal{L}\left(\mathcal{H}^{s-1}(\Omega), \mathcal{H}^{s+1}(\Omega)\right)} \leq\left(K_{r}\right)^{s / r} .
\end{aligned}
$$

Obviously, $K_{r}$ depends on $\Omega$. This dependence is omitted. 
6.2. Regularity of scalar fields. We transpose Theorem 3.1 of [6] to the complex-valued case. Since the second half of the proof (choice of the parameter $k$, here complex-valued; explicit dependence of the regularity exponent $\tau$ and stability constant $c$ on $\mathcal{P}, \xi \ldots)$ is quite different in this case, we provide it for the sake of completeness.

THEOREM 6.8. Let $\xi$ be a scalar, non-constant, complex-valued coefficient that fulfills the coefficient assumption. Then, there exists a regularity exponent $\tau_{\xi}:=$ $\tau\left(\mathcal{P}, \xi_{-} / \xi_{\max }, \Lambda_{\xi}\right) \in\left(0, \frac{1}{2}\right)$ such that, for all $s \in\left[0, \tau_{\xi}\right)$, there exists a constant $c(s, \xi):=c\left(\mathcal{P}, s, \xi_{-} / \xi_{\max }, \Lambda_{\xi}\right)$ such that for all $f \in \mathcal{H}^{s-1}(\Omega)$, the solution $u \in \mathcal{H}^{1}(\Omega)$ to (6.4) belongs to $\mathcal{H}^{s+1}(\Omega)$, and

$$
\|u\|_{\mathcal{H}^{s+1}(\Omega)} \leq \frac{c(s, \xi)}{\xi_{\max }}\|f\|_{\mathcal{H}^{s-1}(\Omega)} .
$$

Remark 6.9. By introducing the scaling factor $\left(\xi_{\max }\right)^{-1}$, one is able to refine the dependence of the constant $c$ on the coefficient, namely that $c$ depends only on the amplitude $\xi_{-} / \xi_{\max }$ and the local, scaled, variations $\Lambda_{\xi}$. And because the coefficient $\xi$ is non-constant, condition (5.1) yields $\xi_{-} / \xi_{\max } \in(0,1)$.

Proof. Let $k \in \mathbb{C} \backslash\{0\}$. Using the operator $D$, we note that given $f^{*} \in \mathcal{H}^{-1}(\Omega)$ and denoting by $u^{*}$ the solution to (6.4) with data $f^{*}$, it holds that

$$
f^{*}=D\left(\xi \nabla u^{*}\right)=D\left(k \nabla u^{*}\right)-D\left((k-\xi) \nabla u^{*}\right)=D\left(\nabla\left(k u^{*}\right)\right)-D\left(\left(1-\frac{\xi}{k}\right) \nabla\left(k u^{*}\right)\right) .
$$

Introducing $\bar{\xi}=(1-\xi / k) \in P W^{1, \infty}(\Omega)$ and $v^{*}=k u^{*} \in \mathcal{H}^{1}(\Omega)$, we get $f^{*}=D\left(\nabla v^{*}\right)-$ $D\left(\bar{\xi} \nabla v^{*}\right)$. Because $L D \nabla$ is equal to the identity operator in $\mathcal{L}\left(\mathcal{H}^{1}(\Omega), \mathcal{H}^{1}(\Omega)\right)$, it follows that

$$
L f^{*}=v^{*}-L\left(D\left(\bar{\xi} \nabla v^{*}\right)\right)=v^{*}-L\left(D\left(m_{\bar{\xi}}\left(\nabla v^{*}\right)\right)\right) .
$$

Let us now denote $Q:=L D m_{\bar{\xi}} \nabla \in \mathcal{L}\left(\mathcal{H}^{1}(\Omega), \mathcal{H}^{1}(\Omega)\right)$. If moreover $Q$ belongs to $\mathcal{L}\left(\mathcal{H}^{s+1}(\Omega), \mathcal{H}^{s+1}(\Omega)\right)$ for some $s \in\left(0, \frac{1}{2}\right)$, then we derive from the above that if we consider some data $f \in \mathcal{H}^{s-1}(\Omega)$ in (6.4), one has

$$
|k|\|u\|_{\mathcal{H}^{s+1}(\Omega)} \leq \frac{\|L\|_{s-1, s+1}}{1-\|Q\|_{s+1, s+1}}\|f\|_{\mathcal{H}^{s-1}(\Omega)},
$$

under the condition $\|Q\|_{s+1, s+1}<1$, where $\|L\|_{s-1, s+1}:=\|L\|_{\mathcal{L}\left(\mathcal{H}^{s-1}(\Omega), \mathcal{H}^{s+1}(\Omega)\right)}$ and $\|Q\|_{s+1, s+1}:=\|Q\|_{\mathcal{L}\left(\mathcal{H}^{s+1}(\Omega), \mathcal{H}^{s+1}(\Omega)\right)}$.

Given $s \in\left(0, \frac{1}{2}\right)$, let $v \in \mathcal{H}^{s+1}(\Omega)$. One checks successively that: $\nabla v \in \boldsymbol{H}^{s}(\Omega)$; $m_{\bar{\xi}}(\nabla v) \in \widetilde{\boldsymbol{H}}^{s}(\Omega)$ (cf. Proposition 6.5); $D\left(m_{\bar{\xi}} \nabla v\right) \in \mathcal{H}^{s-1}(\Omega)$ (cf. Proposition 6.6); $L\left(D m_{\bar{\xi}} \nabla v\right) \in \mathcal{H}^{s+1}(\Omega)$ (cf. Proposition 6.7). In addition, all those results are accompanied by a continuous dependence. Hence, one has $Q \in \mathcal{L}\left(\mathcal{H}^{s+1}(\Omega), \mathcal{H}^{s+1}(\Omega)\right)$, with

$$
\|Q\|_{s+1, s+1} \leq\|L\|_{s-1, s+1}\|D\|_{\mathcal{L}\left(\widetilde{\boldsymbol{H}}^{s}(\Omega), \mathcal{H}^{s-1}(\Omega)\right)}\left\|m_{\bar{\xi}}\right\|_{{\mathcal{L}\left(\boldsymbol{H}^{s}(\Omega), \widetilde{\boldsymbol{H}}^{s}(\Omega)\right)} .}
$$

So it remains to prove that there exists $\tau \in\left(0, \frac{1}{2}\right)$ such that, for all $s \in[0, \tau)$, $\|Q\|_{s+1, s+1}<1$, where the bound on the norm is derived by an appropriate choice of $k \in \mathbb{C} \backslash\{0\}$.

To that aim, let $\tau_{0} \in\left(0, \frac{1}{2}\right)$ be given, and let $s \in\left[0, \tau_{0}\right)$. Using the bound (6.13), we obtain first that

$$
\|Q\|_{s+1, s+1} \leq\left(K_{\tau_{0}}\right)^{s / \tau_{0}} \bar{\xi}_{\text {max }}\left(N_{\bar{\xi}}^{\tau_{0}}\right)^{s / \tau_{0}}
$$


where $\bar{\xi}_{\text {max }}:=\|\bar{\xi}\|_{L^{\infty}(\Omega)}$, according to (6.8), (6.11) and Proposition 6.6. We know from Proposition 6.7 that $K_{\tau_{0}} \geq 1$, and that it is independent of $k$ and $\xi$. Let us study now the behavior of $\bar{\xi}_{\text {max }}$ and $N_{\bar{\xi}}^{\tau_{0}}$ with respect to $k$ and $\xi$, so that for an appropriate choice of $k$ and of $\tau \in\left(0, \tau_{0}\right]$, one can guarantee that

$$
\left(K_{\tau_{0}}\right)^{s / \tau_{0}} \bar{\xi}_{\max }\left(N_{\bar{\xi}}^{\tau_{0}}\right)^{s / \tau_{0}}<1, \quad \forall s \in[0, \tau) .
$$

To achieve (6.15), one needs that $\bar{\xi}_{\text {max }}<1$. Indeed, once $k$ is given, it holds that $\lim _{s \rightarrow 0^{+}}\left(K_{\tau_{0}}\right)^{s / \tau_{0}}\left(N_{\bar{\xi}}^{\tau_{0}}\right)^{s / \tau_{0}}=1$.

Let $k=\bar{\rho} \exp (\imath \widetilde{\theta}), \bar{\rho}>0, \widetilde{\theta} \in[0,2 \pi)$. Keeping the notations of (6.1), one has

$$
|\bar{\xi}(\boldsymbol{x})|^{2}=|1-\xi(\boldsymbol{x}) / k|^{2}=1+\frac{\rho(\boldsymbol{x})}{\bar{\rho}}\left(\frac{\rho(\boldsymbol{x})}{\bar{\rho}}-2 \cos (\theta(\boldsymbol{x})-\widetilde{\theta})\right) \text {, a.e. } \boldsymbol{x} \in \Omega .
$$

It follows that $|\bar{\xi}(\boldsymbol{x})|<1$ a.e. $\boldsymbol{x} \in \Omega$ if, and only if,

$$
\frac{1}{2} \frac{\rho(\boldsymbol{x})}{\bar{\rho}}<\cos (\theta(\boldsymbol{x})-\widetilde{\theta}) \text {, a.e. } \boldsymbol{x} \in \Omega \text {. }
$$

Choosing the angular part $\widetilde{\theta}=\frac{1}{2}\left(\theta_{\min }+\theta_{\max }\right)$ and recalling that $\xi_{\max }=\|\rho\|_{L^{\infty}(\Omega)}$ (cf. (6.1)), this condition is implied by

$$
\frac{1}{2} \frac{\xi_{\max }}{\bar{\rho}}<\cos \left(\frac{1}{2}\left(\theta_{\max }-\theta_{\min }\right)\right) .
$$

Since one has $\theta_{\max }-\theta_{\min } \leq 2 \arccos \left(\xi_{-} / \xi_{\max }\right)$, a sufficient condition is

$$
\frac{1}{2} \frac{\xi_{\max }}{\bar{\rho}}<\frac{\xi_{-}}{\xi_{\max }} \Longleftrightarrow \frac{1}{2} \frac{\left(\xi_{\max }\right)^{2}}{\xi_{-}}<\bar{\rho} .
$$

So, let us choose $k=\gamma\left(\xi_{\max }\right)^{2} / \xi_{-} \exp \left(\frac{\imath}{2}\left(\theta_{\min }+\theta_{\max }\right)\right)$ for some $\gamma \in\left(\frac{1}{2}, \infty\right)$ to be determined. With this value of $k=k(\gamma)$, one can find an upper bound for $\bar{\xi}_{\max }$ :

$$
|\bar{\xi}(\boldsymbol{x})|^{2}=1+\frac{\rho(\boldsymbol{x})\left(\xi_{-}\right)^{2}}{\gamma^{2}\left(\xi_{\max }\right)^{3}}\left(\frac{\rho(\boldsymbol{x})}{\xi_{\max }}-2 \gamma \frac{\xi_{\max }}{\xi_{-}} \cos (\theta(\boldsymbol{x})-\widetilde{\theta})\right) \text {, a.e. } \boldsymbol{x} \in \Omega .
$$

But $\rho(\boldsymbol{x}) \leq \xi_{\max }$ and $\cos (\theta(\boldsymbol{x})-\widetilde{\theta}) \geq \cos \left(\frac{1}{2}\left(\theta_{\max }-\theta_{\min }\right)\right)$ a.e $\boldsymbol{x} \in \Omega$, so

$$
\begin{aligned}
\frac{\rho(\boldsymbol{x})}{\xi_{\max }}-2 \gamma \frac{\xi_{\max }}{\xi_{-}} \cos (\theta(\boldsymbol{x})-\widetilde{\theta}) & \leq 1-2 \gamma \frac{\xi_{\max }}{\xi_{-}} \cos \left(\frac{1}{2}\left(\theta_{\max }-\theta_{\min }\right)\right) \\
& \leq 1-2 \gamma, \text { a.e. } \boldsymbol{x} \in \Omega .
\end{aligned}
$$

Since $1-2 \gamma<0$, we now observe that $\rho(\boldsymbol{x}) \geq \xi_{-}$a.e $\boldsymbol{x} \in \Omega$ leads to

$$
|\bar{\xi}(\boldsymbol{x})|^{2} \leq 1+\frac{1-2 \gamma}{\gamma^{2}}\left(\frac{\xi_{-}}{\xi_{\max }}\right)^{3} \text {, a.e. } \boldsymbol{x} \in \Omega \text {. }
$$

The minimum of $\gamma \mapsto(1-2 \gamma) / \gamma^{2}$ on $\left(\frac{1}{2}, \infty\right)$ is obtained for $\gamma=1$ and is equal to -1 . So we finally choose $k=k(1)$, i.e.

$$
k=\frac{\left(\xi_{\max }\right)^{2}}{\xi_{-}} \exp \left(\frac{\imath}{2}\left(\theta_{\min }+\theta_{\max }\right)\right)
$$


$656\left(1-\left(\frac{\xi_{-}}{\xi_{\max }}\right)^{3}\right)^{1 / 2}\left(2\left(K_{\tau_{0}} D_{\tau_{0}}\right)^{2 / \tau_{0}}\left(1+\frac{C^{2}}{\left(\frac{\xi_{\max }}{\xi_{-}}-1\right)^{2}} \Lambda_{\xi}^{2}\right)\right)^{s / 2}<1, \quad \forall s \in[0, \tau)$.

\footnotetext{
${ }^{1}$ Notice that in the particular case where $\xi$ is piecewise constant, we have that $\Lambda_{\bar{\xi}}=\Lambda_{\xi}=0$.
} 
Since $2\left(K_{\tau_{0}} D_{\tau_{0}}\right)^{2 / \tau_{0}} \geq 2$ (see Proposition 6.7 and $\left.(6.5)\right)$ and $\left(1+C^{2} \cdots\right) \geq 1$, this last condition is equivalent to

$$
\left(1-\left(\frac{\xi_{-}}{\xi_{\max }}\right)^{3}\right)\left(2\left(K_{\tau_{0}} D_{\tau_{0}}\right)^{2 / \tau_{0}}\left(1+\frac{C^{2}}{\left(\frac{\xi_{\max }}{\xi_{-}}-1\right)^{2}} \Lambda_{\xi}^{2}\right)\right)^{\tau}<1 .
$$

This leads to choosing

(6.18)

$$
\tau_{\xi}=\min \left(\tau_{0},-\frac{\log \left(1-\left(\frac{\xi_{-}}{\xi_{\max }}\right)^{3}\right)}{\log 2+\frac{2}{\tau_{0}} \log \left(K_{\tau_{0}} D_{\tau_{0}}\right)+\log \left(1+\frac{C^{2}}{\left(\frac{\xi_{\max }}{\xi_{-}}-1\right)^{2}} \Lambda_{\xi}^{2}\right)}\right) \in\left(0, \tau_{0}\right] .
$$

Finally, we also conclude from (6.12), (6.14), (6.16) and the bounds above that, for all $s \in[0, \tau)$, for all $f \in \mathcal{H}^{s-1}(\Omega)$, the solution $u \in \mathcal{H}^{1}(\Omega)$ to (6.4) belongs to $\mathcal{H}^{s+1}(\Omega)$, and

$$
\begin{aligned}
\|u\|_{\mathcal{H}^{s+1}(\Omega)} & \leq \frac{\xi_{-}}{\left(\xi_{\text {max }}\right)^{2}} \frac{\left(K_{\tau_{0}}\right)^{s / \tau_{0}}}{1-\left(K_{\tau_{0}}\right)^{s / \tau_{0}} \bar{\xi}_{\max }\left(N_{\bar{\xi}_{0}}^{\tau_{0}}\right)^{s / \tau_{0}}}\|f\|_{\mathcal{H}^{s-1}(\Omega)} \\
& \leq \frac{c(s, \xi)}{\xi_{\max }}\|f\|_{\mathcal{H}^{s-1}(\Omega)}, \text { where }
\end{aligned}
$$

$$
c(s, \xi)=\frac{\frac{\xi_{-}}{\xi_{\max }}\left(K_{\tau_{0}}\right)^{s / \tau_{0}}}{1-\left(2\left(K_{\tau_{0}} D_{\tau_{0}}\right)^{2 / \tau_{0}}\left(1+\frac{C^{2}}{\left(\frac{\xi_{\max }}{\xi_{-}}-1\right)^{2}} \Lambda_{\xi}^{2}\right)\right)^{s / 2}\left(1-\left(\frac{\xi_{-}}{\xi_{\text {max }}}\right)^{3}\right)^{1 / 2}} .
$$

This proves the claim.

According to (6.17), one finds that $\bar{\xi}_{\max }=0$ only in the particular case where $\xi_{-}=$ $\xi_{\max }$. As a matter of fact, in this case, we know from (5.1) that $\xi(\boldsymbol{x})=\xi_{-} \exp \left(\imath \theta^{\star}\right)$ a.e. in $\Omega$. Hence, the operator $D m_{\xi} \nabla$ is proportional to the Laplacian $D \nabla$ and the result is trivial: one can even pick any regularity exponent $\tau$ lower than $\frac{1}{2}$, cf. Theorem 6.2.

On the other hand, for a piecewise constant coefficient $\xi$, one has $\Lambda_{\xi}=0$, so that once $\tau_{0} \in\left(0, \frac{1}{2}\right)$ is chosen, (6.18) and (6.19) respectively simplify to:

$$
\begin{aligned}
\tau_{\xi} & =\min \left(\tau_{0},-\frac{\log \left(1-\left(\frac{\xi_{-}}{\xi_{\text {max }}}\right)^{3}\right)}{\log 2+\frac{2}{\tau_{0}} \log \left(K_{\tau_{0}} D_{\tau_{0}}\right)}\right) \in\left(0, \tau_{0}\right] ; \\
c(s, \xi) & =\frac{\frac{\xi_{-}}{\xi_{\text {max }}}\left(K_{\tau_{0}}\right)^{s / \tau_{0}}}{1-\left(2\left(K_{\tau_{0}} D_{\tau_{0}}\right)^{2 / \tau_{0}}\right)^{s / 2}\left(1-\left(\frac{\xi_{-}}{\xi_{\max }}\right)^{3}\right)^{1 / 2}} .
\end{aligned}
$$

For our model problem, of special interest is the electric case, with the coefficient $\xi=\varepsilon_{\sigma}$ : in this case, $\left(\varepsilon_{\sigma}\right)_{-}$can be chosen among $\left(\cos \theta^{\star} \varepsilon_{\min }+\sin \theta^{\star} \sigma_{\min } / \omega\right)_{\theta^{\star} \in[0, \pi / 2]}$. In particular

$$
\left(\varepsilon_{\sigma}\right)_{-} \geq \max _{\theta^{\star} \in[0, \pi / 2]}\left(\cos \theta^{\star} \varepsilon_{m i n}+\sin \theta^{\star} \sigma_{\min } / \omega\right)=\left(\left(\varepsilon_{\min }\right)^{2}+\left(\sigma_{\min } / \omega\right)^{2}\right)^{1 / 2} .
$$


On the other hand,

$$
\left(\varepsilon_{\sigma}\right)_{\max } \leq\left(\left(\varepsilon_{\max }\right)^{2}+\left(\sigma_{\max } / \omega\right)^{2}\right)^{1 / 2} .
$$

When both $\varepsilon$ and $\sigma$ are constant on $\Omega$, the operator $-\operatorname{div} \varepsilon_{\sigma} \nabla$ (with Dirichlet boundary condition) is proportional to the Laplace operator (with Dirichlet boundary condition), and one has $\left(\varepsilon_{\min }\right)^{2}+\left(\sigma_{\min } / \omega\right)^{2}=\left(\varepsilon_{\max }\right)^{2}+\left(\sigma_{\max } / \omega\right)^{2}$, so the bounds on $\left(\varepsilon_{\sigma}\right)_{-}$and $\left(\varepsilon_{\sigma}\right)_{\max }$ are sharp. According again to Theorem 6.2, any $\tau_{\varepsilon_{\sigma}}<\frac{1}{2}$ with $c_{\varepsilon_{\sigma}}=c^{L a p}\left(\tau_{\varepsilon_{\sigma}}\right)$ is admissible in this case.

In the other configurations (non-constant $\varepsilon_{\sigma}$ ), let $\tau_{0} \in\left(0, \frac{1}{2}\right)$ be fixed, and introduce

$$
R_{\varepsilon_{\sigma}}:=\left(\varepsilon_{\sigma}\right)_{\max } /\left(\varepsilon_{\sigma}\right)_{-}>1 ;
$$

then the regularity exponent (6.18), respectively the stability constant (6.19) of Theorem 6.8 , are given by

(6.22) $\tau_{\varepsilon_{\sigma}}=\min \left(\tau_{0},-\frac{\log \left(1-\left(R_{\varepsilon_{\sigma}}\right)^{-3}\right)}{\log 2+\frac{2}{\tau_{0}} \log \left(K_{\tau_{0}} D_{\tau_{0}}\right)+\log \left(1+\frac{C^{2}}{\left(R_{\varepsilon_{\sigma}}-1\right)^{2}} \Lambda_{\varepsilon_{\sigma}}{ }^{2}\right)}\right) \in\left(0, \tau_{0}\right]$

$(6.23) c\left(s, \varepsilon_{\sigma}\right)=\frac{\left(R_{\varepsilon_{\sigma}}\right)^{-1}\left(K_{\tau_{0}}\right)^{s / \tau_{0}}}{1-\left(2\left(K_{\tau_{0}} D_{\tau_{0}}\right)^{2 / \tau_{0}}\left(1+\frac{C^{2}}{\left(R_{\varepsilon_{\sigma}}-1\right)^{2}} \Lambda_{\varepsilon_{\sigma}}{ }^{2}\right)\right)^{s / 2}\left(1-\left(R_{\varepsilon_{\sigma}}\right)^{-3}\right)^{1 / 2}}$.

6.3. Bounding the norm of scalar fields. We now bound the norm of the right-hand sides of the variational formulations (5.4) and (5.7), governing resp. $p_{0}$ and $q_{0}$.

LEMma 6.10. Let $\Omega$ be a domain such that (Top) $I$ is fulfilled, and assume that $\xi$ fulfills the coefficient assumption. Let $\boldsymbol{v} \in \boldsymbol{X}_{B}(\Omega, \xi)$ be given, for $B \in\{$ Dir, Neu $\}$. Let $s \in\left[0, \frac{1}{2}\right):$ if $B=$ Dir, the right-hand sides $f$ defined by (5.4) belong to $\mathcal{H}_{\text {Dir }}^{s-1}(\Omega)$; resp. if $B=$ Neu the right-hand sides $f$ defined by (5.7) belong to $\mathcal{H}_{N e u}^{s-1}(\Omega)$. In addition, for all $s \in\left(0, \frac{1}{2}\right)$, it holds that

$$
\|f\|_{\mathcal{H}^{s-1}(\Omega)} \leq \mathrm{c}^{B} s^{1 / 2}\|\operatorname{div} \xi \boldsymbol{v}\|_{0, \Omega}+C_{\left(s, \frac{1}{2}\right)} \xi_{\max } N_{\xi}^{s}\|\boldsymbol{v}\|_{\boldsymbol{H}(\mathbf{c u r l} ; \Omega)} .
$$

Proof. Let us focus on the case $B=\operatorname{Dir}$ (proof is similar in the case $B=N e u$ ). We use the same notations as in Theorem 5.5. Introduce $f \in \mathcal{H}^{-1}(\Omega)$ :

$$
f: \psi \mapsto\left(\xi\left(\boldsymbol{z}+\boldsymbol{v}_{r e g}\right) \mid \nabla \psi\right)_{0, \Omega}+(\operatorname{div} \xi \boldsymbol{v} \mid \psi)_{0, \Omega}, \quad \forall \psi \in \mathcal{H}^{1}(\Omega) .
$$

Let $s \in\left(0, \frac{1}{2}\right)$ be given.

First, it is obvious that $f_{0}: \psi \mapsto(\operatorname{div} \xi \boldsymbol{v} \mid \psi)_{0, \Omega}$ belongs to $\mathcal{H}^{s-1}(\Omega)$. Indeed, according to Appendix A:

$\forall \psi \in \mathcal{H}^{1-s}(\Omega),\left|(\operatorname{div} \xi \boldsymbol{v} \mid \psi)_{0, \Omega}\right| \leq\|\operatorname{div} \xi \boldsymbol{v}\|_{0, \Omega}\|\psi\|_{0, \Omega} \leq \mathrm{c}^{\operatorname{Dir}} s^{1 / 2}\|\operatorname{div} \xi \boldsymbol{v}\|_{0, \Omega}\|\psi\|_{\mathcal{H}^{1-s}(\Omega)}$. Hence, $f_{0} \in \mathcal{H}^{s-1}(\Omega)$ and $\left\|f_{0}\right\|_{\mathcal{H}^{s-1}(\Omega)} \leq \mathrm{c}^{\text {Dir }} s^{1 / 2}\|\operatorname{div} \xi \boldsymbol{v}\|_{0, \Omega}$. Then we recall that $\boldsymbol{z}+\boldsymbol{v}_{\text {reg }}$ belongs to $\boldsymbol{H}^{\frac{1}{2}}(\Omega) \subset \boldsymbol{H}^{s}(\Omega)$, so that $m_{\xi}\left(\boldsymbol{z}+\boldsymbol{v}_{\text {reg }}\right)=$ $\xi\left(\boldsymbol{z}+\boldsymbol{v}_{\text {reg }}\right) \in \widetilde{\boldsymbol{H}}^{s}(\Omega)$ according to Proposition 6.5. Then it follows from Proposition 6.6 that $D\left(m_{\xi}\left(\boldsymbol{z}+\boldsymbol{v}_{r e g}\right)\right) \in \mathcal{H}^{s-1}(\Omega)$. In other words, $\psi \mapsto\left(\xi\left(\boldsymbol{z}+\boldsymbol{v}_{r e g}\right) \mid \nabla \psi\right)_{0, \Omega}$ also belongs to $\mathcal{H}^{s-1}(\Omega)$.

Regarding the norm estimate (6.24), we simply use the bounds (6.7) and (6.9) together with (5.3) to conclude the proof: the dependence in $s$ (the constant $\left.C_{\left(s, \frac{1}{2}\right)}\right)$ comes from the continuous embedding $\boldsymbol{H}^{\frac{1}{2}}(\Omega) \subset \boldsymbol{H}^{s}(\Omega)$. 
Given $r \in\left(0, \frac{1}{2}\right)$, one may use (6.8) for $s \in(0, r]$, and thus replace (6.24) for all $s \in(0, r]$ by

$$
\|f\|_{\mathcal{H}^{s-1}(\Omega)} \leq \mathrm{c}^{B} s^{1 / 2}\|\operatorname{div} \xi \boldsymbol{v}\|_{0, \Omega}+C_{\left(s, \frac{1}{2}\right)} \xi_{\text {max }}\left(N_{\xi}^{r}\right)^{s / r}\|\boldsymbol{v}\|_{\boldsymbol{H}(\mathbf{c u r l} ; \Omega)} .
$$

6.4. Application to the polyhedral model problem. We recall that, under the assumptions defining the polyhedral model problem, the coefficients $(\varepsilon, \mu, \sigma)$ are such that $\varepsilon, \sigma$ fulfill the coefficient assumption, and $\mu$ is constant on $\Omega$. According to the definition of the model problem (2.9), it always hold that $\mu^{-1}$ curl $\boldsymbol{e} \in$ $\boldsymbol{X}_{N e u}(\Omega, \mu)$. Here, because $\mu$ is a constant, $\boldsymbol{X}_{N e u}(\Omega, \mu)=\boldsymbol{X}_{N e u}(\Omega)$. It follows that $\mu^{-1}$ curl $\boldsymbol{e} \in \boldsymbol{H}^{\sigma_{N e u}}(\Omega)$ with $\sigma_{N e u}>\frac{1}{2}$, cf. Proposition 5.3. Then, regarding the choice of a regularity exponent for the electric field $\boldsymbol{e}$ itself, because $\varepsilon_{\sigma} \in P W^{1, \infty}(\Omega)$, we note that either any $\tau_{\varepsilon_{\sigma}}<\frac{1}{2}$ is admissible (constant $\varepsilon_{\sigma}$ ), or that it is given by (6.22) (non-constant $\varepsilon_{\sigma}$ ). Indeed, one has the regular/gradient splitting (6.2):

$$
\boldsymbol{e}=\boldsymbol{e}_{r e g}+\boldsymbol{z}_{e}+\nabla p_{0} \text { in } \Omega \text {, where } \boldsymbol{e}_{r e g} \in \boldsymbol{H}^{1}(\Omega), \boldsymbol{z}_{e} \in \boldsymbol{H}^{\sigma_{D i r}}(\Omega) .
$$

The regularity of the gradient part, namely $\nabla p_{0} \in \boldsymbol{H}^{s}(\Omega)$ is a straightforward consequence of Theorems 6.2 (constant $\varepsilon_{\sigma}$ ) and 6.8 (non-constant $\varepsilon_{\sigma}$ ), provided that the right-hand side $f$ given there belongs to $\mathcal{H}^{s-1}(\Omega)$, for all values $s \in\left[0, \tau_{\varepsilon_{\sigma}}\right)$. But, since this regularity result on $f$ was proven in Lemma 6.10, one has indeed $\nabla p_{0} \in \boldsymbol{H}^{s}(\Omega)$, for all $s \in\left[0, \tau_{\varepsilon_{\sigma}}\right)$.

It follows that we can provide values for $\tau_{(\varepsilon, \mu, \sigma)}$ (regularity exponent), resp. $C_{(\varepsilon, \mu, \sigma, s)}^{\star}$ (stability constant), in (3.6). Because the limiting value of a regularity exponent is constrained by $\tau_{\varepsilon_{\sigma}}\left(\tau_{\varepsilon_{\sigma}}<\frac{1}{2}<\sigma_{N e u}\right)$, we choose

$$
\tau_{(\varepsilon, \mu, \sigma)}:=\tau_{\varepsilon_{\sigma}} \in\left(0, \frac{1}{2}\right) .
$$

From now on, we assume that we are given a set $\Theta$ of coefficients $(\varepsilon, \mu, \sigma)$, such that $\varepsilon, \sigma$ fulfill the coefficient assumption, and $\mu$ is constant on $\Omega$. Moreover, we consider the case where $\underline{\tau}:=\inf _{(\varepsilon, \mu, \sigma) \in \Theta} \tau_{(\varepsilon, \mu, \sigma)}>0$. This covers in particular the case where $\Theta$ is a singleton $\left\{\left(\varepsilon^{0}, \mu^{0}, \sigma^{0}\right)\right\}$, and $\underline{\tau}=\tau_{\left(\varepsilon^{0}, \mu^{0}, \sigma^{0}\right)}$.

Lemma 6.11. Let $p_{0}$ be defined as in (5.4). For all $(\varepsilon, \mu, \sigma) \in \Theta$, for all $s \in(0, \underline{\tau})$, the norm in $\mathcal{H}^{s-1}(\Omega)$ of the right-hand side $f$ in (5.4) is bounded by

$$
\begin{aligned}
& \|f\|_{\mathcal{H}^{s-1}(\Omega)} \leq c_{0}^{0} \omega\left\|\boldsymbol{j}_{\text {ext }}\right\|_{0, \Omega}+c_{\mathrm{div}}^{0} \omega^{-1}\left\|\operatorname{div} \boldsymbol{j}_{\text {ext }}\right\|_{0, \Omega}, \text { where } \\
& c_{0}^{0}:=C_{\left(s, \frac{1}{2}\right)}\left(\varepsilon_{\sigma}\right)_{\max }\left(N_{\bar{\varepsilon}_{\sigma}}\right)^{s / \underline{\tau}}\left(C_{(\varepsilon, \mu, \sigma)}^{c o e r}\right)^{-1}, \text { and } c_{\mathrm{div}}^{0}:=\mathrm{c}^{D i r} s^{1 / 2} .
\end{aligned}
$$

Remark 6.12. We recall that

$$
\left(N_{\varepsilon_{\sigma}}^{\frac{\tau}{}}\right)^{s / \underline{\tau}}:=\left(D_{\underline{\tau}}\right)^{s / \underline{\tau}}\left(2\left(1+C^{2} \Lambda_{\varepsilon_{\sigma}}{ }^{2}\right)\right)^{s / 2},
$$

where $D_{\tau}$ is defined at (6.5), resp. $C$ is the Poincaré constant defined at (6.6), and $\Lambda_{\varepsilon_{\sigma}}:=\left(\left(\varepsilon_{\sigma}\right)_{\max }\right)^{-1}\left|\varepsilon_{\sigma}\right|_{P W^{1, \infty}(\Omega)}$. Note that only $D_{\underline{\underline{\tau}}}$ and $C$, and hence $N \bar{\varepsilon}_{\sigma}^{\tau}$, depend on the partition induced by $(\varepsilon, \mu, \sigma)$.

Proof. According to (6.25), we know that the right-hand side that defines $\nabla p_{0}$ is bounded by

$$
\|f\|_{\mathcal{H}^{s-1}(\Omega)} \leq \mathrm{c}^{D i r} s^{1 / 2}\left\|\operatorname{div} \varepsilon_{\sigma} \boldsymbol{e}\right\|_{0, \Omega}+C_{\left(s, \frac{1}{2}\right)}\left(\varepsilon_{\sigma}\right)_{\max }\left(N_{\mathcal{E}_{\sigma}}^{\frac{\tau}{}}\right)^{s / \underline{\tau}}\|\boldsymbol{e}\|_{\boldsymbol{H}(\mathbf{c u r l} ; \Omega)} .
$$


Going back to the model problem (2.9), the left part of the upper bound is readily replaced by $c^{D i r} s^{1 / 2} \omega^{-1}\left\|\operatorname{div} j_{e x t}\right\|_{0, \Omega}$. Then, using the coercivity bound of Proposition 4.1:

$$
C_{(\varepsilon, \mu, \sigma)}^{\text {coer }}\|\boldsymbol{e}\|_{\boldsymbol{H}(\mathbf{c u r l} ; \Omega)}^{2} \leq \omega\left|\left(\boldsymbol{j}_{\text {ext }} \mid \boldsymbol{e}\right)_{0, \Omega}\right| \leq \omega\left\|\boldsymbol{j}_{\text {ext }}\right\|_{0, \Omega}\|\boldsymbol{e}\|_{\boldsymbol{H}(\mathbf{c u r l} ; \Omega)},
$$

we find for the right part the bound

$$
C_{\left(s, \frac{1}{2}\right)}\left(\varepsilon_{\sigma}\right)_{\max }\left(N_{\mathcal{\varepsilon}_{\sigma}}^{\frac{\tau}{s}}\right)^{s / \underline{\tau}}\left(C_{(\varepsilon, \mu, \sigma)}^{c o e r}\right)^{-1} \omega\left\|\boldsymbol{j}_{\text {ext }}\right\|_{0, \Omega},
$$

which proves the claim.

The bound on $\left\|\nabla p_{0}\right\|_{s, \Omega}=\left\|p_{0}\right\|_{\mathcal{H}^{1+s}(\Omega)}$ follows: for all $s \in(0, \underline{\tau})$,

$$
\left\|\nabla p_{0}\right\|_{s, \Omega} \leq \frac{c_{D i r}\left(s, \varepsilon_{\sigma}\right)}{\left(\varepsilon_{\sigma}\right)_{\max }}\left(c_{0}^{0} \omega\left\|\boldsymbol{j}_{e x t}\right\|_{0, \Omega}+c_{\mathrm{div}}^{0} \omega^{-1}\left\|\operatorname{div} \boldsymbol{j}_{e x t}\right\|_{0, \Omega}\right),
$$

where $c_{D i r}\left(s, \varepsilon_{\sigma}\right)$ is given in Theorem 6.2 or Theorem $6.8 ; c_{D i r}\left(s, \varepsilon_{\sigma}\right)$ depends on the partition induced by $(\varepsilon, \mu, \sigma)$.

We are now in a position to estimate the norm of $\boldsymbol{e}$ in $\boldsymbol{P} \boldsymbol{H}^{s}(\mathbf{c u r l} ; \Omega)$, for all values $s \in(0, \underline{\tau})$, which then leads to the desired convergence rate.

Lemma 6.13. For all $(\varepsilon, \mu, \sigma) \in \Theta$, for all $s \in(0, \underline{\tau})$, one has the estimate

$$
\begin{aligned}
& \|\boldsymbol{e}\|_{\boldsymbol{P} \boldsymbol{H}^{s}(\mathbf{c u r l} ; \Omega)} \leq C_{0(\varepsilon, \mu, \sigma, s)}^{\star}\left\|\boldsymbol{j}_{\text {ext }}\right\|_{0, \Omega}+C_{\operatorname{div}(\varepsilon, \mu, \sigma, s)}^{\star}\left\|\operatorname{div} \boldsymbol{j}_{\text {ext }}\right\|_{0, \Omega} \text {, where }
\end{aligned}
$$

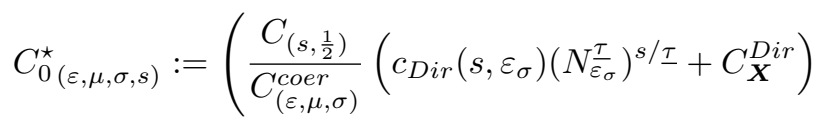$$
\left.+C_{\left(s, \sigma_{N e u}\right)} I_{\boldsymbol{X}_{N e u}}\left(\frac{\left(1+\mu \omega^{2}\left(\varepsilon_{\sigma}\right)_{\max }\right)}{C_{(\varepsilon, \mu, \sigma)}^{c o e r}}+\mu\right)\right) \omega
$$

$$
C_{\operatorname{div}(\varepsilon, \mu, \sigma, s)}^{\star}:=\frac{c_{D i r}\left(s, \varepsilon_{\sigma}\right) \mathrm{c}^{D i r} s^{1 / 2}}{\left(\varepsilon_{\sigma}\right)_{\max }} \omega^{-1},
$$

and $I_{\boldsymbol{X}_{N e u}}$ denotes the norm of the embedding $\boldsymbol{X}_{N e u}(\Omega) \subset \boldsymbol{H}^{\sigma_{N e u}}(\Omega)$.

Remark 6.14. The above is slightly different from (3.6), where both contributions of the norm $\left\|\boldsymbol{j}_{\text {ext }}\right\|_{\boldsymbol{H}(\mathrm{div} ; \Omega)}$ are merged. Also, only $c_{D i r}\left(s, \varepsilon_{\sigma}\right)$ and $N_{\varepsilon_{\sigma}}$ depend on the partition induced by $(\varepsilon, \mu, \sigma)$.

Proof. One has $\|\boldsymbol{e}\|_{s, \Omega} \leq\left\|\boldsymbol{e}_{r e g}+\boldsymbol{z}_{e}\right\|_{s, \Omega}+\left\|\nabla p_{0}\right\|_{s, \Omega}$, and the bound on $\left\|\nabla p_{0}\right\|_{s, \Omega}$ is given right above. On the other hand,

$$
\begin{aligned}
\left\|\boldsymbol{e}_{r e g}+\boldsymbol{z}_{e}\right\|_{s, \Omega} & \leq C_{\left(s, \frac{1}{2}\right)}\left\|\boldsymbol{e}_{r e g}+\boldsymbol{z}_{e}\right\|_{1 / 2, \Omega} \\
& \leq C_{\left(s, \frac{1}{2}\right)} C_{\boldsymbol{X}}^{D i r}\|\boldsymbol{e}\|_{\boldsymbol{H}(\mathbf{c u r l} ; \Omega)} \leq \frac{C_{\left(s, \frac{1}{2}\right)} C_{\boldsymbol{X}}^{D i r}}{C_{(\varepsilon, \mu, \sigma)}^{\text {coer }}} \omega\left\|\boldsymbol{j}_{e x t}\right\|_{0, \Omega} .
\end{aligned}
$$

We conclude that: for all $s \in(0, \underline{\tau})$,

$\|\boldsymbol{e}\|_{s, \Omega} \leq\left(\frac{c_{D i r}\left(s, \varepsilon_{\sigma}\right) c_{0}^{0}}{\left(\varepsilon_{\sigma}\right)_{\max }}+\frac{C_{\left(s, \frac{1}{2}\right)} C_{\boldsymbol{X}}^{\text {Dir }}}{C_{(\varepsilon, \mu, \sigma)}^{c o e r}}\right) \omega\left\|\boldsymbol{j}_{\text {ext }}\right\|_{0, \Omega}+\frac{c_{D i r}\left(s, \varepsilon_{\sigma}\right) c_{\mathrm{div}}^{0} \omega^{-1}}{\left(\varepsilon_{\sigma}\right)_{\max }}\left\|\operatorname{div} \boldsymbol{j}_{\text {ext }}\right\|_{0, \Omega}$,

where $c_{0}^{0}$ and $c_{\mathrm{div}}^{0}$ are defined in Lemma 6.11.

Regarding the norm of curl $\boldsymbol{e}$, we recall that curl $\boldsymbol{e} \in \boldsymbol{X}_{N e u}(\Omega)$. But $\boldsymbol{X}_{N e u}(\Omega)$ is 
continuously embedded in $\boldsymbol{H}^{\sigma_{N e u}}(\Omega)$ (Proposition 5.3) so we find that for all $s \in(0, \underline{\tau})$,

$$
\begin{aligned}
\|\operatorname{curl} e\|_{s, \Omega} & \leq C_{\left(s, \sigma_{N e u}\right)}\|\operatorname{curl} \boldsymbol{e}\|_{\sigma_{N e u}, \Omega} \\
& \leq C_{\left(s, \sigma_{N e u}\right)} I_{\boldsymbol{X}_{N e u}}\|\operatorname{curl} \boldsymbol{e}\|_{\boldsymbol{X}_{\text {Neu }}(\Omega)} \\
& =C_{\left(s, \sigma_{\text {Neu }}\right)} I_{\boldsymbol{X}_{\text {Neu }}}\|\operatorname{curl} \boldsymbol{e}\|_{\boldsymbol{H}(\operatorname{curl} ; \Omega) .}
\end{aligned}
$$

Next, we have, using the model problem (2.9):

$$
\begin{aligned}
\|\operatorname{curl} \boldsymbol{e}\|_{\boldsymbol{H}(\operatorname{curl} ; \Omega)} & \leq\|\operatorname{curl} \boldsymbol{e}\|_{0, \Omega}+\|\operatorname{curl} \operatorname{curl} \boldsymbol{e}\|_{0, \Omega} \\
& =\|\operatorname{curl} \boldsymbol{e}\|_{0, \Omega}+\mu\left\|\operatorname{curl} \mu^{-1} \operatorname{curl} \boldsymbol{e}\right\|_{0, \Omega} \\
& =\|\operatorname{curl} \boldsymbol{e}\|_{0, \Omega}+\mu\left\|\omega^{2} \varepsilon_{\sigma} \boldsymbol{e}+\imath \omega \boldsymbol{j}_{\text {ext }}\right\|_{0, \Omega} \\
& \leq\left(1+\mu \omega^{2}\left(\varepsilon_{\sigma}\right)_{\max }\right)\|\boldsymbol{e}\|_{\boldsymbol{H}(\operatorname{curl} ; \Omega)}+\mu \omega\left\|\boldsymbol{j}_{\text {ext }}\right\|_{0, \Omega} \\
& \leq\left(\frac{\left(1+\mu \omega^{2}\left(\varepsilon_{\sigma}\right)_{\max }\right)}{C_{(\varepsilon, \mu, \sigma)}^{\operatorname{coer}}}+\mu\right) \omega\left\|\boldsymbol{j}_{\text {ext }}\right\|_{0, \Omega} .
\end{aligned}
$$

Hence, for all $s \in(0, \underline{\tau})$,

$$
\|\operatorname{curl} \boldsymbol{e}\|_{s, \Omega} \leq C_{\left(s, \sigma_{N e u}\right)} I_{\boldsymbol{X}_{N e u}}\left(\frac{\left(1+\mu \omega^{2}\left(\varepsilon_{\sigma}\right)_{\max }\right)}{C_{(\varepsilon, \mu, \sigma)}^{c o e r}}+\mu\right) \omega\left\|\boldsymbol{j}_{e x t}\right\|_{0, \Omega}
$$

Then, using Proposition 3.1, we find, for all $s \in(0, \underline{\tau})$ :

$$
\|\boldsymbol{e}\|_{\boldsymbol{P H}^{s}(\operatorname{curl} ; \Omega)} \leq\|\boldsymbol{e}\|_{\boldsymbol{P} \boldsymbol{H}^{s}(\Omega)}+\|\operatorname{curl} \boldsymbol{e}\|_{\boldsymbol{P} \boldsymbol{H}^{s}(\Omega)} \leq\|\boldsymbol{e}\|_{s, \Omega}+\|\operatorname{curl} \boldsymbol{e}\|_{s, \Omega}
$$

and the conclusion follows.

We recall that $C_{(\varepsilon, \mu, \sigma)}^{\sharp}=C_{(\varepsilon, \mu, \sigma)}^{c c o t}\left(C_{(\varepsilon, \mu, \sigma)}^{c o e r}\right)^{-1}$ is the constant appearing in Céa's lemma (and bounded in $\S 4$ ) and that $\varsigma>0$ is the shape regularity parameter of the family of meshes. Then one has the following convergence rate for the polyhedral model problem.

THEOREM 6.15. Let $(\varepsilon, \mu, \sigma)$ be such that $\varepsilon, \sigma$ fulfill the coefficient assumption, and $\mu$ is constant on $\Omega$. For all $s \in\left(0, \tau_{(\varepsilon, \mu, \sigma)}\right)$, there exist constants $C_{(\varsigma, s)}^{\text {interp }}$, $C_{0(\varepsilon, \mu, \sigma, s)}^{\star}$, and $C_{\operatorname{div}(\varepsilon, \mu, \sigma, s)}^{\star}$ such that for all $\boldsymbol{j}_{\text {ext }} \in \boldsymbol{H}(\operatorname{div} ; \Omega)$ and all $h$, the error estimate hold:

$$
\begin{aligned}
\left\|\boldsymbol{e}-\boldsymbol{e}_{h}\right\|_{\boldsymbol{H}(\mathbf{c u r l} ; \Omega)} \leq C_{(\varepsilon, \mu, \sigma)}^{\sharp} C_{(\varsigma, s)}^{\text {interp }} h^{s} & \left(C_{0(\varepsilon, \mu, \sigma, s)}^{\star}\left\|\boldsymbol{j}_{\text {ext }}\right\|_{0, \Omega}\right. \\
& \left.+C_{\operatorname{div}(\varepsilon, \mu, \sigma, s)}^{\star}\left\|\operatorname{div} \boldsymbol{j}_{\text {ext }}\right\|_{0, \Omega}\right) .
\end{aligned}
$$

Let $\Theta$ be a set of coefficients $(\varepsilon, \mu, \sigma)$ whose elements are all piecewise smooth on the same polyhedral partition, and assume that $\underline{\tau}:=\inf _{(\varepsilon, \mu, \sigma) \in \Theta} \tau_{(\varepsilon, \mu, \sigma)}>0$. Then (6.26) holds true for all $s \in(0, \underline{\tau})$.

Remark 6.16. The above is slightly different from (3.7), where both contributions of the norm $\left\|\boldsymbol{j}_{\text {ext }}\right\|_{\boldsymbol{H}(\mathrm{div} ; \Omega)}$ are merged.

Proof. It is straightforward to derive the result (6.26) by using successively (3.3) and (3.5), and finally the estimate of Lemma 6.13 for $\underline{\tau}=\tau_{(\varepsilon, \mu, \sigma)}$. 
6.5. A few possible generalizations. Let us mention two cases we have excluded so far: first when $\mu$ fulfills the coefficient assumption, but $\mu$ is not constant on $\Omega$; second when $\boldsymbol{j}_{\text {ext }} \notin \boldsymbol{H}(\operatorname{div} ; \Omega)$, but $\boldsymbol{j}_{\text {ext }} \in \boldsymbol{L}^{2}(\Omega)$ with $\operatorname{div} \boldsymbol{j}_{\text {ext }} \in H^{-t}(\Omega)$ for some $t \in(0,1)$.

In the first situation, all the previous analyses apply, except when one addresses the regularity of $\mu^{-1}$ curl $\boldsymbol{e}$ with respect to the scale $\left(\boldsymbol{H}^{s}(\Omega)\right)_{s}$. Although it still holds that $\mu^{-1}$ curl $\boldsymbol{e} \in \boldsymbol{X}_{N e u}(\Omega, \mu)$, one has $\boldsymbol{X}_{N e u}(\Omega, \mu) \neq \boldsymbol{X}_{N e u}(\Omega)$. To find a regularity exponent, one uses now (6.3), where the regularity is determined by the gradient part $\nabla q_{0}$ : see Theorem 6.8 (Neumann case), which yields the value of the regularity exponent $\tau_{\mu}$. One then chooses

$$
\tau_{(\varepsilon, \mu, \sigma)}:=\min \left(\tau_{\varepsilon_{\sigma}}, \tau_{\mu}\right) \in\left(0, \frac{1}{2}\right) .
$$

Next, one derives an estimate on $\|\operatorname{curl} e\|_{s, \Omega}$, for all $s \in\left(0, \tau_{(\varepsilon, \mu, \sigma)}\right)$. Noting that the multiplicative operator $m_{\mu}$ belongs to $\mathcal{L}\left(\boldsymbol{H}^{s}(\Omega), \boldsymbol{H}^{s}(\Omega)\right)$, it follows that

$$
\|\operatorname{curl} \boldsymbol{e}\|_{s, \Omega} \leq\left\|m_{\mu}\right\|_{\mathcal{L}\left(\boldsymbol{H}^{s}(\Omega), \boldsymbol{H}^{s}(\Omega)\right)}\left\|\mu^{-1} \operatorname{curl} \boldsymbol{e}\right\|_{s, \Omega} .
$$

The first quantity, $\left\|m_{\mu}\right\|_{\mathcal{L}\left(\boldsymbol{H}^{s}(\Omega), \boldsymbol{H}^{s}(\Omega)\right)}$, is easily bounded from above, thanks to Proposition 6.5 and (6.5).

Then, using (6.3), one writes

$$
\left\|\mu^{-1} \operatorname{curl} \boldsymbol{e}\right\|_{s, \Omega} \leq\left\|\boldsymbol{c}_{r e g}+\boldsymbol{z}_{c}\right\|_{s, \Omega}+\left\|\nabla q_{0}\right\|_{s, \Omega} .
$$

Thanks to (5.6), one has

$$
\left\|\boldsymbol{c}_{r e g}+\boldsymbol{z}_{c}\right\|_{s, \Omega} \leq C_{\left(s, \frac{1}{2}\right)}\left\|\boldsymbol{c}_{r e g}+\boldsymbol{z}_{c}\right\|_{1 / 2, \Omega} \leq C_{\left(s, \frac{1}{2}\right)} C_{\boldsymbol{X}}^{N e u}\left\|\mu^{-1} \operatorname{curl} \boldsymbol{e}\right\|_{\boldsymbol{H}(\operatorname{curl} ; \Omega)} .
$$

On the other hand, according to Theorem 6.8 it holds that

$$
\left\|\nabla q_{0}\right\|_{s, \Omega} \leq \frac{c_{N e u}(s, \mu)}{\mu_{\max }}\|f\|_{\mathcal{H}^{s-1}(\Omega)},
$$

where $f$ is the right-hand side of (5.7). Using Lemma 6.10 yields

$$
\left\|\nabla q_{0}\right\|_{s, \Omega} \leq c_{N e u}(s, \mu) C_{\left(s, \frac{1}{2}\right)}\left(N_{\mu}^{\frac{\tau}{\mu}}\right)^{s / \underline{\tau}}\left\|\mu^{-1} \operatorname{curl} \boldsymbol{e}\right\|_{\boldsymbol{H}(\operatorname{curl} ; \Omega)} .
$$

Aggregating the two estimates, one finds now

$$
\left\|\mu^{-1} \operatorname{curl} \boldsymbol{e}\right\|_{s, \Omega} \leq C_{\left(s, \frac{1}{2}\right)}\left(C_{\boldsymbol{X}}^{N e u}+c_{N e u}(s, \mu)\left(N \frac{\tau}{\mu}\right)^{s / \underline{\tau}}\right)\left\|\mu^{-1} \operatorname{curl} \boldsymbol{e}\right\|_{\boldsymbol{H}(\mathbf{c u r l} ; \Omega)} .
$$

Writing finally

$$
\begin{aligned}
\left\|\mu^{-1} \operatorname{curl} \boldsymbol{e}\right\|_{\boldsymbol{H}(\operatorname{curl} ; \Omega)} & \leq\left\|\mu^{-1} \operatorname{curl} \boldsymbol{e}\right\|_{0, \Omega}+\left\|\operatorname{curl} \mu^{-1} \operatorname{curl} \boldsymbol{e}\right\|_{0, \Omega} \\
& \leq \frac{1}{\mu_{\min }}\|\operatorname{curl} \boldsymbol{e}\|_{0, \Omega}+\left\|\operatorname{curl} \mu^{-1} \operatorname{curl} \boldsymbol{e}\right\|_{0, \Omega} \\
(\operatorname{cf.}(2.9)) & \leq\left(\frac{1}{\mu_{\min }}+\omega^{2}\left(\varepsilon_{\sigma}\right)_{\max }\right)\|\boldsymbol{e}\|_{\boldsymbol{H}(\operatorname{curl} ; \Omega)}+\omega\left\|\boldsymbol{j}_{\text {ext }}\right\|_{0, \Omega}
\end{aligned}
$$

and using the coercivity, the rest of the estimates follow easily. 
In the second situation, namely when $\operatorname{div} \boldsymbol{j}_{\text {ext }} \in H^{-t}(\Omega)$ for some $t \in(0,1)$, one must use a generalized regular/gradient splitting. Precisely, one introduces (see $[4$, Theorem 6.1.15]):

$$
\boldsymbol{X}_{D i r}(\Omega, \xi,-t):=\left\{\boldsymbol{v} \in \boldsymbol{H}_{0}(\operatorname{curl} ; \Omega): \operatorname{div} \xi \boldsymbol{v} \in H^{-t}(\Omega)\right\} .
$$

Then, one may generalize Theorem 5.5 to elements of $\boldsymbol{X}_{D i r}(\Omega, \xi,-t)$. the only difference is that $\nabla p_{0}$ is now governed by

$$
\left\{\begin{array}{l}
\text { Find } p_{0} \in H_{0}^{1}(\Omega) \text { such that } \\
\left(\xi \nabla p_{0} \mid \nabla \psi\right)_{0, \Omega}=-(\xi \boldsymbol{z} \mid \nabla \psi)_{0, \Omega}-\left(\xi \boldsymbol{v}_{r e g} \mid \nabla \psi\right)_{0, \Omega}-\langle\operatorname{div} \xi \boldsymbol{v}, \psi\rangle_{H_{0}^{t}(\Omega)}, \forall \psi \in H_{0}^{1}(\Omega) .
\end{array}\right.
$$

With this result at hand, one may proceed as before, replacing the occurences of $\left\|\operatorname{div} \boldsymbol{j}_{\text {ext }}\right\|_{0, \Omega}$ by $\left\|\operatorname{div} \boldsymbol{j}_{\text {ext }}\right\|_{-t, \Omega}$ : one simply notices that when $\operatorname{div} \boldsymbol{j}_{\text {ext }} \in H^{-t}(\Omega)$, one may still apply Lemma 6.10 , but only for all $s \in\left(0, \min \left(\frac{1}{2}, 1-t\right)\right)$. Hence, one chooses

$$
\tau_{(\varepsilon, \mu, \sigma, t)}:=\min \left(\tau_{\varepsilon_{\sigma}}, \tau_{\mu}, 1-t\right) \in\left(0, \frac{1}{2}\right) .
$$

Computations can then be carried out.

Then, what happens when $\Theta$ is not reduced to a singleton? For simplicity ${ }^{2}$, let us consider that all its elements $(\varepsilon, \mu, \sigma)$ are such that $\varepsilon, \sigma, \mu$ are piecewise-constant on a fixed partition and that $\boldsymbol{j}_{\text {ext }} \in \boldsymbol{H}(\operatorname{div} ; \Omega)$. Then the regularity exponent writes

$$
\underline{\tau}:=\inf _{(\varepsilon, \mu, \sigma) \in \Theta} \min \left(\tau_{\varepsilon_{\sigma}}, \tau_{\mu}\right) .
$$

According to (6.20), we see that if condition

$$
\sup _{(\varepsilon, \mu, \sigma) \in \Theta} \frac{\left(\varepsilon_{\sigma}\right)_{\max }}{\left(\varepsilon_{\sigma}\right)_{-}}+\sup _{(\varepsilon, \mu, \sigma) \in \Theta} \frac{\mu_{\max }}{\mu_{\min }}<\infty
$$

holds, then $\underline{\tau}>0$ and for any $(\varepsilon, \mu, \sigma) \in \Theta$ one may apply the previous results for all $s \in[0, \underline{\tau})$. Note that this condition is comparable to the one found using the local approach, see (B.8), because one has $\left(\varepsilon_{\sigma}\right)_{\max } \leq \omega^{-1}\left(\varepsilon_{\max }^{2} \omega^{2}+\sigma_{\max }^{2}\right)^{1 / 2}$, and $\left(\varepsilon_{\sigma}\right)_{-} \geq \omega^{-1}\left(\varepsilon_{\min }^{2} \omega^{2}+\sigma_{\min }^{2}\right)^{1 / 2}$. On the other hand, there exist configurations such that if, eg., $\sup _{(\varepsilon, \mu, \sigma) \in \Theta} \mu_{\max } / \mu_{\min }=\infty$, it holds that $\inf _{(\varepsilon, \mu, \sigma) \in \Theta} \tau_{\mu}=0$. In other words, there is no (extra-)regularity in this limit case. We refer to $\S 7.2$ for an illustration.

Also, what can be said in the context of $\S 5.3$, that is when $\xi$ is a complex-valued, measurable, tensor field that fulfills the coefficient assumption? It turns out that one may address the case of a normal tensor field, ie. $\xi=\mathbb{U}^{-1} \mathbb{D} U$ a.e. in $\Omega$, where $\mathbb{U}$ is a unitary tensor field, resp. $\mathbb{D}$ is a diagonal tensor field, defined in $\Omega$. Let us briefly explain why.

First, Proposition 6.5 still applies. Then, (5.1) is now replaced by (5.8): but the latter can be seen as the equivalent of the former, imposed on $\mathbb{D}_{11}, \mathbb{D}_{22}$ and $\mathbb{D}_{33}$. Hence one may use the reformulated (6.1), namely

$$
\mathbb{D}_{k k} \in\left\{z=\rho \exp (\imath \theta), \rho \in\left[\xi_{-}, \xi_{\text {max }}\right], \theta \in\left[\theta_{\text {min }}, \theta_{\text {max }}\right]\right\} \text { a.e. in } \Omega \text {, for } k=1,2,3 \text {, }
$$

$$
\text { where } \xi_{\text {max }}:=\sup _{\boldsymbol{z} \in \mathbb{C}^{3} \backslash\{0\}} \operatorname{ess}_{\Omega} \sup _{\Omega} \frac{\mid \xi \boldsymbol{z}]}{|\boldsymbol{z}|} \text {, and } 0 \leq \theta_{\max }-\theta_{\min } \leq 2 \arccos \left(\frac{\xi_{-}}{\xi_{\max }}\right) \text {, }
$$

\footnotetext{
2 If more generally the coefficients are piecewise smooth, or if the partition depends on the element $(\varepsilon, \mu, \sigma)$, the condition is more involved than (6.27) proposed below.
} 
in the proof of Theorem 6.8.

The proof then proceeds as before, and one can conclude that Theorem 6.8 still holds. The rest of the proofs are unchanged.

Finally, let us mention that the non-conductive case $(\sigma=0)$ can be handled similarly, under the assumption that the model problem is well-posed (ie. $\omega^{2}$ is not an eigenvalue of the corresponding eigenproblem). In this case the coercivity constant is frequency dependent, in the sense that it is inversely proportional to the distance of $\omega^{2}$ to the closest eigenvalue, see for instance $[4, \S 8.3]$. On the other hand, the estimates on the regularity exponent and on the stability constant can still be recovered in this context.

7. Evaluating the regularity exponent. Below, we evaluate the "sharpness" of the bounds on $\tau_{(\varepsilon, \mu, \sigma)}$ on two examples.

7.1. The coplanar waveguide. First, let us consider the coplanar waveguide case, as provided by the MORwiki Community [34, 5]. Precisely, the geometry of interest is a parallelepided, see Figure 1. The upper part of the domain is made of air, while the bottom part is made of a substrate (in yellow) in which three perfectly conducting striplines (in blue) are embedded. The electric permittivity $\varepsilon$ and the conductivity $\sigma$ are piecewise constant (with different values in the air and in the substrate), while the magnetic permeability $\mu$ takes the same value in the air and in the substrate.

The resulting $\Omega$ is thus equal to the paralleliped minus the three striplines, and the interface $\Sigma$ separates the two materials (air, substrate): it is flat, see Figure 1. Importantly, all angles, either at the boundary, or at the interface between the two materials, are equal to mutiples of $\pi / 2$. One solves the model problem (2.9) in this configuration for a given $\boldsymbol{j}_{\text {ext }}$.

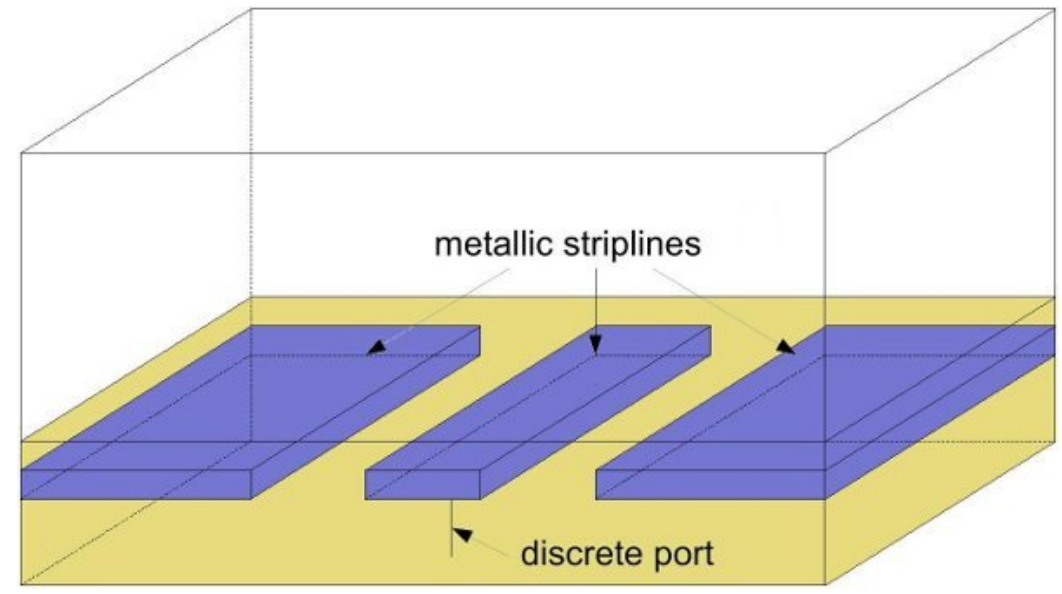

FIG. 1. A coplanar waveguide [34].

Since the coefficients are piecewise constant, one may use the local approach (cf. Appendix B). According to the framework developed there, one has to study the local problems on interior domains (no intersection with $\partial \Omega$, cf. $\S \mathrm{B} .1$ ) resp. on boundary domains (cf. $\S \mathrm{B} .2)$, to determine the "best regularity" exponent $\tau_{(\varepsilon, \mu, \sigma)}^{o p t}$. In the 
present case, studying local problems on interior domains, see (B.7), one finds that there is no constraint on $\tau_{(\varepsilon, \mu, \sigma)}^{\text {opt }}$ because the interface is flat. On the other hand, the study of local problems on boundary domains is more involved a priori; there are edge problems with interface (at the intersection $\partial \Omega \cap \Sigma$ ), edge problems without interface (on $\partial \Omega$ ), and finally corner problems without interface (on $\partial \Omega$ ).

Interestingly, for the edge problems with interface and because the diedric angles are equal to $\pi / 2$ in both materials, one notices that, using a symmetry argument (odd reflection for the Dirichlet boundary condition, resp. even reflection for the Neumann boundary condition, see [22, p. 41]), one can recast the problem as an interior problem, with a flat interface. Hence, $\tau_{\mathbf{e}, \Sigma}(\xi)=1$ for $\xi \in\left\{\mu, \varepsilon_{\sigma}\right\}$.

Also, making the same observation on the value of the angles, and using again a symmetry argument, one can recast the corner problem without interface as an edge problem without interface: it follows that $\tau_{\mathrm{c}, \partial \Omega}(\xi)=\tau_{\mathbf{e}, \partial \Omega}(\xi)$, where $\tau_{\mathbf{e}, \partial \Omega}(\xi)$ is characterized next, for $\xi \in\left\{\mu, \varepsilon_{\sigma}\right\}$, and one has $\tau_{(\varepsilon, \mu, \sigma)}^{o p t}=\min \left(1, \tau_{\mathrm{e}, \partial \Omega}(\mu), \tau_{\mathrm{e}, \partial \Omega}\left(\varepsilon_{\sigma}\right)\right)$.

So, in the end, there remains to study the edge problem without interface to determine the value of $\tau_{(\varepsilon, \mu, \sigma)}^{\text {opt }}$. But this is a standard problem: one looks for the regularity exponent in an L-shape (local) domain $\mathcal{O}$ for the Laplace operator (there is no interface, hence no jump of the coefficient) with either homogeneous Dirichlet or, homogeneous Neumann, boundary condition: it is well-known that $\tau_{\mathrm{e}, \partial \Omega}(\mu)=\tau_{\mathrm{e}, \partial \Omega}\left(\varepsilon_{\sigma}\right)=2 / 3$. Aggregating all results, one concludes that $\tau_{(\varepsilon, \mu, \sigma)}^{o p t}=2 / 3$ for the coplanar waveguide, independently of the values of the coefficients $(\varepsilon, \mu, \sigma)$. In other words the lower bounds provided by (6.27) and (B.8) are not "sharp".

7.2. The checkerboard. We study now a simple example (cf. for instance Dauge's benchmark [16]), to illustrate the fact that the conditions (6.27) and (B.8) can be "sharp": let us consider the domain $\Omega:=(-1,1) \times(-1,1) \times(0,1)$, made of four cubes, stacked together:

$$
\begin{array}{ll}
\Omega_{1}:=(-1,0) \times(-1,0) \times(0,1), & \Omega_{2}:=(0,1) \times(-1,0) \times(0,1), \\
\Omega_{3}:=(0,1) \times(0,1) \times(0,1), & \Omega_{4}:=(-1,0) \times(0,1) \times(0,1) .
\end{array}
$$

We assume that $\varepsilon$ and $\sigma$ are constant, while $\mu$ is piecewise constant, and equal to 1 in $\Omega_{1} \cup \Omega_{3}$, resp. to $\delta \in(0,1)$ in $\Omega_{2} \cup \Omega_{4}$.

Again, all angles are multiples of $\pi / 2$. Proceeding as in $\S 7.1$, one observes first that $\tau_{(\varepsilon, \mu, \sigma)}^{o p t}=\min \left(1, \tau_{\mathbf{e}, \Sigma}(\mu)\right)$. Then, solving (B.7) and looking for the smallest non-zero eigenvalue $\nu_{0}$, one may check that it is governed by

$$
\cos \left(\sqrt{\nu_{0}} \frac{\pi}{2}\right)=\frac{1-\delta}{1+\delta}, \text { or equivalently } \sqrt{\nu_{0}}=\frac{2}{\pi} \arccos \left(\frac{1-\delta}{1+\delta}\right) .
$$

Performing the expansion in the limit $\delta \rightarrow 0$, one finds that $\sqrt{\nu_{0}} \sim \frac{4}{\pi} \sqrt{\delta}$. Since $\tau_{\mathrm{e}, \Sigma(\mu)}=\sqrt{\nu_{0}}$, we find $\tau_{(\varepsilon, \mu, \sigma)}^{\text {opt }} \sim \frac{4}{\pi} \sqrt{\delta}$, so there is no (extra-)regularity in the limit case: in this sense the conditions (6.27) and (B.8) are "sharp" for the checkerboard. To conclude, we provide some excerpts from Dauge's benchmark [16], see Table 1. These numerical values corroborate the asymptotic formula when $\delta$ goes to 0 .

7.3. Comments. When applicable, the local approach allows one to compute the "best" regularity exponent $\tau_{(\varepsilon, \mu, \sigma)}^{o p t}$, up to numerical precision. As a matter of fact, one has to solve numerically a series of eigenproblems, see $\S \S B .1-B .2$. Or, in more favorable cases (cf. §7.1), it is even known exactly. However, the theory we recalled in Appendix B is limited to the case of piecewise constant coefficients, on a polyhedral 


\begin{tabular}{|c|c|c|}
\hline$\delta$ & $\frac{4}{\pi} \sqrt{\delta}$ & $\left(\sqrt{\nu_{0}}\right)_{\text {computed }}$ \\
\hline $10^{-1}$ & $4.026310^{-1}$ & $3.899610^{-1}$ \\
\hline $10^{-2}$ & $1.273210^{-1}$ & $1.269010^{-1}$ \\
\hline $10^{-8}$ & $1.273210^{-4}$ & $1.273210^{-4}$ \\
\hline
\end{tabular}

TABLE 1

Asymptotic and computed values of $\sqrt{\nu_{0}}$.

partition. Or, at least, to coefficients that are locally (piecewise) constant near the interface and locally smooth near the boundary. And, near the boundary and for smooth coefficients, one may use the so-called frozen coefficients technique, cf. [21, $\S 5.2]$ or $[17, \S 5]$. In principle, the value of $\tau_{(\varepsilon, \mu, \sigma)}^{\text {opt }}$ can still be computed. But when the coefficients are only piecewise smooth, the technique no longer applies.

Also, there is no obvious way to compute the constant $C_{(\varepsilon, \mu, \sigma, t)}^{\star}$ appearing in (3.6) when $t$ spans $\left(0, \tau_{(\varepsilon, \mu, \sigma)}^{o p t}\right)$, or to provide bounds of such a constant with respect to the coeffficients, with the help of the local approach.

On the other hand, the global approach allows one to address all of the above, on a partition made of (possibly) non-polyhedral domains. It is only when the discretization is concerned that one assumes the partition to be made of polyhedra.

8. Acknowledgements. The author thanks Julia Charrier for many interesting discussions and feedback, and Erell Jamelot and Serge Nicaise for providing some useful insight regarding the local approach (Appendix B). The author also acknowledges the contribution of Vasileios Vrettakos, who analyzed the coplanar waveguide configuration during his Master's internship [35] using different mathematical tools and measures of electromagnetic fields than the ones presented here.

\section{REFERENCES}

[1] M. Ainsworth, W. McLean, and T. Tran. The conditioning of boundary element equations on locally refined meshes and preconditioning by diagonal scaling. SIAM J. Numer. Anal., 36:1901-1932, 1999.

[2] A. Alonso and A. Valli. An optimal domain decomposition preconditioner for low-frequency time harmonic Maxwell equations. Math. Comp., 68:607-631, 1999.

[3] C. Amrouche, C. Bernardi, M. Dauge, and V. Girault. Vector potentials in three-dimensional non-smooth domains. Math. Meth. Appl. Sci., 21:823-864, 1998.

[4] F. Assous, P. Ciarlet, Jr., and S. Labrunie. Mathematical foundations of computational electromagnetism, volume 198 of Applied Mathematical Sciences. Springer, 2018.

[5] P. Benner and J. Schneider. Uncertainty quantification for Maxwell's equations using stochastic collocation and model order reduction. International Journal for Uncertainty Quantification, 5:195-208, 2015.

[6] A. Bonito, J.-L. Guermond, and F. Luddens. Regularity of the Maxwell equations in heterogeneous media and Lipschitz domains. J. Math. Anal. Appl., 408:498-512, 2013.

[7] A.-S. Bonnet-Ben Dhia, L. Chesnel, and P. Ciarlet, Jr. T-coercivity for the Maxwell problem with sign-changing coefficients. Communications in PDEs, 39:1007-1031, 2014.

[8] A.-S. Bonnet-Ben Dhia, L. Chesnel, and P. Ciarlet, Jr. Two-dimensional Maxwell's equations with sign-changing coefficients. Appl. Numer. Math., 79:29-41, 2014.

[9] S. Brenner and L.R. Scott. The mathematical theory of finite element methods, 3rd Edition. Springer Verlag, 2008.

[10] M. Campos Pinto and B. Després. Constructive formulations of resonant Maxwell's equations. SIAM J. Math. Anal., 49:3637-3670, 2017.

[11] S.N. Chandler-Wilde, D.P. Hewett, and A. Moiola. Interpolation of Hilbert and Sobolev spaces: quantitative estimates and counterexamples. Mathematika, 61:414-443, 2015.

[12] P. Ciarlet, Jr. On the approximation of electromagnetic fields by edge finite elements. Part 1 : 
Sharp interpolation results for low-regularity fields. Comput. Math. Appl., 71:85-104, 2016.

[13] P. Ciarlet, Jr., S. Fliss, and C. Stohrer. On the approximation of electromagnetic fields by edge finite elements. Part 2: A heterogeneous multiscale method for Maxwell's equations. Comput. Math. Appl., 73:1900-1919, 2017.

[14] M. Costabel, M. Dauge, and S. Nicaise. Singularities of Maxwell interface problems. Math. Mod. Num. Anal., 33:627-649, 1999.

[15] M. Costabel, M. Dauge, and S. Nicaise. Singularities of eddy current problems. Math. Mod. Num. Anal., 37:807-831, 2003.

[16] M. Dauge. Benchmark computations for Maxwell equations. https://perso.univ-rennes1.fr/ monique.dauge/core/index.html.

[17] M. Dauge. Neumann and mixed problems on curvilinear polyhedra. Integr. Equat. Oper. Th., 15:227-261, 1992.

[18] B. Després, L.-M. Imbert-Gérard, and R. Weder. Hybrid resonance of Maxwell's equations in slab geometry. J. Math. Pures Appl., 101:623-659, 2014.

[19] A. Ern and J.-L. Guermond. Finite element quasi-interpolation and best approximation. ESAIM: M2AN, 51:1367-1385, 2017.

[20] A. Ern and J.-L. Guermond. Analysis of the edge finite element approximation of the Maxwell equations with low regularity solutions. Comput. Math. Appl., 75:918-932, 2018.

[21] P. Grisvard. Elliptic problems in nonsmooth domains. Pitman, 1985.

[22] P. Grisvard. Singularities in boundary value problems, volume 22 of RMA. Masson, 1992.

[23] P.W. Gross and P.R. Kotiuga. Electromagnetic theory and computation: a topological approach. MSRI Publications Series, 48. Cambridge University Press, 2004.

[24] N. Heuer. Additive Schwarz method for the p-version of the boundary element method for the single layer potential operator on a plane screen. Numer. Math., 88:485-511, 2001.

[25] N. Heuer. On the equivalence of fractional-order Sobolev semi-norms. J. Math. Anal. Appl., 417:505-518, 2014.

[26] D. S. Jerison and C. E. Kenig. The inhomogeneous Dirichlet problem in Lipschitz domains. J. Functional Analysis, 130:161-219, 1995.

[27] F. Jochmann. An $H^{s}$-regularity result for the gradient of solutions to elliptic equations with mixed boundary conditions. J. Math. Anal. Appl., 238:429-450, 1999.

[28] J.-L. Lions and E. Magenes. Problèmes aux limites non homogènes et applications. Dunod, 1968.

[29] P. Monk. Finite element methods for Maxwell's equations. Oxford University Press, 2003.

[30] J.-C. Nédélec. Mixed finite elements in $\mathbb{R}^{3}$. Numer. Math., 35:315-341, 1980.

[31] J.-C. Nédélec. Acoustic and Electromagnetic Equations. Springer, 2001.

[32] G. Savaré. Regularity results for elliptic equations in Lipschitz domains. J. Funct. Anal., 152:176-201, 1998.

[33] L. Tartar. An introduction to Sobolev spaces and interpolation spaces, volume 3 of Lecture Notes of the Unione Matematica Italiana. Springer, 2007.

[34] The MORwiki Community. Coplanar waveguide. MORwiki - Model Order Reduction Wiki, 2018.

[35] V. Vrettakos. Quantification de l'incertain en électromagnétisme : identification des dépendances par rapport aux paramètres. Master's thesis, Paris-Saclay University, Palaiseau, France, 2016.

Appendix A. Real interpolation method. We follow here [33, §§22-23] and $[9, \S 14]$. Let $H_{0}, H_{1}$ be two Hilbert spaces, continuously embedded into a third Hilbert space $H$ :

- $H_{0}+H_{1}$ is equipped with the norm $\|v\|_{H_{0}+H_{1}}=\inf _{v=v_{0}+v_{1}}\left(\left\|v_{0}\right\|_{H_{0}}+\left\|v_{1}\right\|_{H_{1}}\right)$;

- resp., $H_{0} \cap H_{1}$ is equipped with the norm $\|v\|_{H_{0} \cap H_{1}}=\max \left(\|v\|_{H_{0}},\|v\|_{H_{1}}\right)$.

One introduces, for $v \in H_{0}+H_{1}, K(t ; v):=\inf _{v=v_{0}+v_{1}}\left(\left\|v_{0}\right\|_{H_{0}}^{2}+t^{2}\left\|v_{1}\right\|_{H_{1}}^{2}\right)^{1 / 2}$. For $s \in(0,1)$, one defines the interpolated space

$$
\left(H_{0}, H_{1}\right)_{s, 2}:=\left\{v \in H_{0}+H_{1} \text { s.t. } t^{-s} K(t ; v) \in L^{2}\left(0, \infty ; \frac{d t}{t}\right)\right\},
$$

equipped with the norm $\|v\|_{\left(H_{0}, H_{1}\right)_{s, 2}}:=\left\|t^{-s} K(t ; v)\right\|_{L^{2}\left(0, \infty ; \frac{d t}{t}\right)}$.

In the case where $H_{1} \subset H_{0}$, we use the notation $H_{s}=\left(H_{0}, H_{1}\right)_{s, 2}$. In this case and after elementary computations, one finds that: 
- For all $s, s^{\prime} \in(0,1), s \leq s^{\prime}$ :

$$
\exists C_{\left(s, s^{\prime}\right)}, \forall v \in H_{s^{\prime}}, \quad\|v\|_{H_{s}} \leq C_{\left(s, s^{\prime}\right)}\|v\|_{H_{s^{\prime}}} .
$$

- For all $s \in(0,1), H_{s} \subset H_{0}$ :

$$
\exists \mathrm{c}_{0}>0, \forall s \in(0,1), \forall v \in H_{s},\|v\|_{H_{0}} \leq \mathrm{c}_{0} s^{1 / 2}(1-s)^{1 / 2}\|v\|_{H_{s}} .
$$

- For all $s \in(0,1), H_{1} \subset H_{s}$ :

$$
\exists \mathrm{c}_{1}>0, \forall s \in(0,1), \forall v \in H_{1}, s^{1 / 2}(1-s)^{1 / 2}\|v\|_{H_{s}} \leq \mathrm{c}_{1}\|v\|_{H_{1}} .
$$

If $H_{1}=H_{0}$, the above holds for $c_{0}=2$, resp. $c_{1}=\frac{1}{\sqrt{2}}$.

Appendix B. The local approach for finding a regularity exponent $\tau_{(\varepsilon, \mu, \sigma)}$. We know that $\boldsymbol{e}$ and $\mu^{-1} \operatorname{curl} \boldsymbol{e}$ may be split into regular and gradient parts (6.2)-(6.3). The regularity of the regular parts is known to be independent of the coefficients (see Proposition 5.3). On the other hand, the regularity of the gradient parts, $\nabla p_{0}$ governed by (5.4), resp. $\nabla q_{0}$ governed by (5.7), depends a priori on the coefficients: it is now determined by the local approach. Based on the global approach, we have obtained a regularity exponent $\tau_{(\varepsilon, \mu, \sigma)}$ which is strictly lower than $\frac{1}{2}$. So it may not be equal to the "best" regularity exponent $\tau_{(\varepsilon, \mu, \sigma)}^{o p t}$. Indeed, one knows that the best regularity exponent is always lower than or equal to 1 , and that there exist configurations for which it is equal to 1 , see eg. [3]. To summarize, $\tau_{(\varepsilon, \mu, \sigma)}^{\text {opt }} \in(0,1]$ and

- either $p_{0}, q_{0} \in P H^{2}(\Omega)$ always holds, in which case $\tau_{(\varepsilon, \mu, \sigma)}^{\text {opt }}=1$;

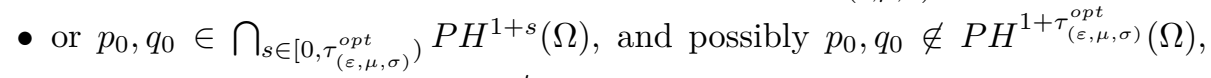
always holds, in which case $\tau_{(\varepsilon, \mu, \sigma)}^{o p t} \in(0,1)$.

Below, we recall how one can characterize the best regularity exponent $\tau_{(\varepsilon, \mu, \sigma)}^{\text {opt }}$ by the local approach, in the case where the coefficients $(\varepsilon, \mu, \sigma)$ are as in section 2.1, and moreover $(\varepsilon, \mu, \sigma)$ are piecewise constant over a polyhedral partition. Below, we focus on the influence of the interface $\Sigma$, that is to say on the influence of the coefficients. Obviously, the influence of the boundary $\partial \Omega$ must be taken into account. But unless the interface intersects with it, the coefficients do not play a role in the regularity of the solution there, only the geometry of the boundary does. Along the same lines, if the coefficient is constant on $\Omega$, the local approach allows one to determine the "best" (largest) value of $\sigma_{D i r}$ and $\sigma_{N e u}$. We follow here $[14,15]$ and Refs. therein: according to Kondratiev's theory, one studies the second order elliptic PDEs locally all over $\Omega$, and in particular the (local) regularity of its solution [21, §8.2].

B.1. Interior domain. Let $\mathcal{O}$ be the domain on which a local problem is defined. We consider here that $\mathcal{O}$ is an interior domain, that is $\partial \mathcal{O} \cap \partial \Omega=\emptyset$. The case of a boundary domain $(\partial \mathcal{O} \cap \partial \Omega \neq \emptyset)$ is sketched in $\S$ B.2.

The first type of local problems occurs when $\overline{\mathcal{O}}$ does not intersect with the interface, and then the solution belongs to $H^{2}(\mathcal{O})$.

There are three other types of local problems, namely:

- problems where the interface is a smooth manifold, in this case the solution is piecewise smooth, ie. it belongs to $P H^{2}(\mathcal{O})$;

- edge problems where the interface is a smooth manifold, except for one edge e, that allow to determine the so-called edge singularities: there exists $\tau_{\mathbf{e}} \in(0,1)$ such that the solution always belongs to $\bigcap_{s \in\left[0, \tau_{\mathrm{e}}\right)} P H^{1+s}(\mathcal{O})$, but may not belong to $P H^{1+\tau_{e}}(\mathcal{O})$; 
- corner problems, where the interface is smooth, except for several edges that intersect at a corner $\mathrm{c}$, that allow to determine the so-called corner singularities: there exists $\tau_{c} \in(0,1)$ such that the solution always belongs to $\bigcap_{s \in\left[0, \tau_{c}\right)} P H^{1+s}(\mathcal{O})$, but may not belong to $P H^{1+\tau_{c}}(\mathcal{O})$.

We focus first on corner problems. The case of the edge problem is treated next. For a corner c, the singularities are obtained as non-zero quasi-homogeneous functions which solve the same problem in $\Gamma$ with zero right-hand side, where $\Gamma$ is the infinite cone that coincides with the domain $\mathcal{O}$ at c. Introducing $\mathbb{S}^{2}$ the unit sphere, resp. $(\rho, \theta, \varphi) \in \mathbb{R}^{+} \times[0, \pi) \times[0,2 \pi)$ the spherical coordinates, centered at $\mathbf{c}$, and defining $G:=\Gamma \cap \mathbb{S}^{2}$, one can choose a priori those functions in the sets

$$
S_{\mathrm{c}}^{\lambda}(\Gamma):=\left\{\Psi=\rho^{\lambda} \psi(\theta, \varphi) \text { s.t. } \psi \in H^{1}(G)\right\}, \text { where } \lambda \in \mathbb{C} .
$$

More precisely (see [15, p. 818]), one should look for quasi-homogeneous functions of the type $\rho^{\lambda} \sum_{q=0, Q}(\log \rho)^{q} \psi_{q}(\theta, \varphi)$ with $Q \in \mathbb{N}$. However it is sufficient for our purposes - determining the exponent - to focus on homogeneous functions. In spherical coordinates, we recall that the volume element writes $\rho^{2} d \rho d \varsigma$, where $d \varsigma:=\sin \theta d \theta d \varphi$, whereas the gradient writes $\nabla v=\partial_{\rho} v \boldsymbol{e}_{\rho}+\rho^{-1} \nabla_{\varsigma} v$, with $\nabla_{\varsigma} v:=$ $\partial_{\theta} v \boldsymbol{e}_{\theta}+(\sin \theta)^{-1} \partial_{\varphi} v \boldsymbol{e}_{\varphi}$.

Because $\mathcal{O}$ is an interior domain, observe that one has $G=\mathbb{S}^{2}$ and $\partial \Gamma=\emptyset$.

For the local corner problem at hand, since one is looking for $\Psi_{c}=\rho^{\lambda_{c}} \psi(\theta, \varphi)$ in $H_{l o c}^{1}(\Gamma)$, one finds that a necessary and sufficient condition on the exponent $\lambda_{c}$ is that $\Re\left(\lambda_{c}\right)>-\frac{1}{2}$; and moreover that

$$
\tau_{\mathrm{c}}(\xi):=\min _{\lambda_{\mathrm{c}} \text { st. } \Re\left(\lambda_{c}\right)>-\frac{1}{2}}\left(\lambda_{\mathrm{c}}+\frac{1}{2}\right),
$$

where $\Psi_{\mathrm{c}} \neq 0$ is a (non-smooth) function governed by $\operatorname{div}\left(\xi \nabla \Psi_{\mathrm{c}}\right)=0$ in $\Gamma$. The coefficient $\xi$ being independent of $\rho$, one easily checks that it is equivalent to finding solutions to the eigenproblem

$$
\left\{\begin{array}{l}
\text { Find } \psi \in H^{1}\left(\mathbb{S}^{2}\right) \backslash\{0\}, \nu \in \mathbb{C} \text { such that } \\
\int_{\mathbb{S}^{2}} \xi \nabla_{\varsigma} \psi \cdot \nabla_{\varsigma} \overline{\psi^{\prime}} d \varsigma=\nu \int_{\mathbb{S}^{2}} \xi \psi \overline{\psi^{\prime}} d \varsigma, \forall \psi^{\prime} \in H^{1}\left(\mathbb{S}^{2}\right),
\end{array}\right.
$$

with the relation $\nu=\lambda_{c}\left(\lambda_{c}+1\right)$. Note that $\psi=1$ and $\nu=0$ is an eigenpair of (B.1), which yields the values $\lambda_{c}=0$ or $\lambda_{c}=-1$. The latter is excluded, because one has necessarily $\Re\left(\lambda_{c}\right)>-\frac{1}{2}$. Whereas the former yields $\Psi_{c}=1$, which is a smooth function, and thus one concludes that no singular behavior is associated with $\nu=0$. Then, choosing $\psi^{\prime}=\psi$ in (B.1) one finds that

$$
\int_{\mathbb{S}^{2}} \xi\left|\nabla_{\varsigma} \psi\right|^{2} d \varsigma=\nu \int_{\mathbb{S}^{2}} \xi|\psi|^{2} d \varsigma .
$$

Using the notation $v_{R}:=\Re(v)$ and $v_{I}:=\Im(v)$ for complex-valued fields, and taking respectively the real and imaginary part of the previous equation, one derives the relations

$$
\begin{aligned}
\nu_{R} & :=\frac{\left(\int_{\mathbb{S}^{2}} \xi_{R}\left|\nabla_{\varsigma} \psi\right|^{2} d \varsigma\right)\left(\int_{\mathbb{S}^{2}} \xi_{R}|\psi|^{2} d \varsigma\right)+\left(\int_{\mathbb{S}^{2}} \xi_{I}\left|\nabla_{\varsigma} \psi\right|^{2} d \varsigma\right)\left(\int_{\mathbb{S}^{2}} \xi_{I}|\psi|^{2} d \varsigma\right)}{\left(\int_{\mathbb{S}^{2}} \xi_{R}|\psi|^{2} d \varsigma\right)^{2}+\left(\int_{\mathbb{S}^{2}} \xi_{I}|\psi|^{2} d \varsigma\right)^{2}} \\
\nu_{I} & :=\frac{\left(\int_{\mathbb{S}^{2}} \xi_{I}\left|\nabla_{\varsigma} \psi\right|^{2} d \varsigma\right)\left(\int_{\mathbb{S}^{2}} \xi_{R}|\psi|^{2} d \varsigma\right)-\left(\int_{\mathbb{S}^{2}} \xi_{R}\left|\nabla_{\varsigma} \psi\right|^{2} d \varsigma\right)\left(\int_{\mathbb{S}^{2}} \xi_{I}|\psi|^{2} d \varsigma\right)}{\left(\int_{\mathbb{S}^{2}} \xi_{R}|\psi|^{2} d \varsigma\right)^{2}+\left(\int_{\mathbb{S}^{2}} \xi_{I}|\psi|^{2} d \varsigma\right)^{2}} .
\end{aligned}
$$


Considering both cases $\xi=\mu$ and $\xi=\varepsilon_{\sigma}$, one notices that, under the assumptions of section 2.1, the spectral theorem can be applied to characterize the solutions to the eigenproblem (B.1), see for instance [31, §2.1]. In particular, the eigenfunctions can be chosen as the elements of a Hilbert basis of $L^{2}\left(\mathbb{S}^{2}\right)$. We already observed that $\psi=1$ is an eigenfunction (with related eigenvalue $\nu=0$ ). Hence, as a consequence of the Poincaré inequality in $H_{z m v}^{1}\left(\mathbb{S}^{2}\right)$, there exists $c_{P}>0$ such that, for all eigenfunctions $\psi$ related to a non-zero eigenvalue, it holds that

$$
\frac{\int_{\mathbb{S}^{2}}\left|\nabla_{\varsigma} \psi\right|^{2} d \varsigma}{\int_{\mathbb{S}^{2}}|\psi|^{2} d \varsigma} \geq c_{P}^{2}
$$

For the two cases of interest:

- If $\xi=\mu$ (real-valued coefficient case), one has according to (B.2)-(B.3) that

$$
\nu=\frac{\int_{\mathbb{S}^{2}} \mu\left|\nabla_{\varsigma} \psi\right|^{2} d \varsigma}{\int_{\mathbb{S}^{2}} \mu|\psi|^{2} d \varsigma} \geq \frac{\mu_{\min }}{\mu_{\max }} c_{P}^{2}>0 .
$$

Recall that $\nu=\lambda_{c}\left(\lambda_{c}+1\right)$, ie. $\lambda_{c}=-\frac{1}{2} \pm \sqrt{\nu+\frac{1}{4}}$. Due to the condition $\Re\left(\lambda_{c}\right)>-\frac{1}{2}$ and because one has $\nu>0$, the only admissible relation is $\lambda_{\mathrm{c}}=-\frac{1}{2}+\sqrt{\nu+\frac{1}{4}}$. Hence $\lambda_{\mathrm{c}}>0$ for all non-zero eigenvalues $\nu$, which yields $\tau_{c}(\mu)>1 / 2$ independently of the values of $\mu_{\min }$ and $\mu_{\max }$.

- If $\xi=\varepsilon_{\sigma}$ (complex-valued coefficient case), one has according to (B.2) that

$$
\nu_{R} \geq \frac{\left(\xi_{R}\right)_{\min }^{2}+\left(\xi_{I}\right)_{\min }^{2}}{\left(\xi_{R}\right)_{\max }^{2}+\left(\xi_{I}\right)_{\max }^{2}} \frac{\int_{\mathbb{S}^{2}}\left|\nabla_{\varsigma} \psi\right|^{2} d \varsigma}{\int_{\mathbb{S}^{2}}|\psi|^{2} d \varsigma} \geq \frac{\varepsilon_{\min }^{2} \omega^{2}+\sigma_{\min }^{2}}{\varepsilon_{\max }^{2} \omega^{2}+\sigma_{\max }^{2}} c_{P}^{2}>0
$$

while, according to (B.3), $\nu_{I}$ can take positive or negative values. Due to the condition $\Re\left(\lambda_{c}\right)>-\frac{1}{2}$ and because one has now $\nu_{R}>0$, see (B.6), the only admissible relation is again $\lambda_{c}=-\frac{1}{2}+\sqrt{\nu+\frac{1}{4}}$. Let $\nu+\frac{1}{4}=\rho_{\nu} \exp \left(\imath \theta_{\nu}\right)$, $\rho_{\nu}>0, \theta_{\nu} \in[0,2 \pi)$. Then $\Re\left(\sqrt{\nu+\frac{1}{4}}\right)=\left(\rho_{\nu}\right)^{1 / 2} \cos \left(\theta_{\nu} / 2\right)$, with $\rho_{\nu} \geq\left(\nu_{R}+\frac{1}{4}\right)$ and $\cos ^{2}\left(\theta_{\nu} / 2\right)=\frac{1}{2}\left(1+\cos \theta_{\nu}\right)=\frac{1}{2}\left(1+\left(\rho_{\nu}\right)^{-1}\left(\nu_{R}+\frac{1}{4}\right)\right)$. Hence,

$$
\Re\left(\sqrt{\nu+\frac{1}{4}}\right)=\left(\frac{1}{2}\left(\rho_{\nu}+\nu_{R}+\frac{1}{4}\right)\right)^{1 / 2} \geq\left(\nu_{R}+\frac{1}{4}\right)^{1 / 2}>\frac{1}{2} .
$$

This yields $\tau_{\mathrm{c}}\left(\varepsilon_{\sigma}\right)>1 / 2$ independently of the values of $\varepsilon_{\max }, \varepsilon_{\min }, \sigma_{\max }$ and $\sigma_{\text {min }}$.

We focus now on edge problems. For an edge e, the singularities are obtained as non-zero quasi-homogeneous functions which solve the same problem in $\Gamma$ with zero right-hand side, where $\Gamma \times \mathbb{R}$ is the infinite sector that coincides with the domain $\mathcal{O}$ at e. Introducing $\mathbb{S}^{1}$ the unit circle, resp. $(\rho, \theta, z) \in \mathbb{R}^{+} \times[0,2 \pi) \times \mathbb{R}$ the cylindrical coordinates with e $\subset\{z=0\}$, and defining $G:=\Gamma \cap \mathbb{S}^{1}$, one can choose a priori those functions in the sets

$$
S_{\mathrm{e}}^{\lambda}(\Gamma):=\left\{\Psi=\rho^{\lambda} \psi(\theta) \text { s.t. } \psi \in H^{1}(G)\right\} \text {, where } \lambda \in \mathbb{C} .
$$


One should look for quasi-homogeneous functions, however restricting to homogeneous is again sufficient to determine the exponent. In the polar coordinates $(\rho, \theta)$, we recall that the surface element writes $\rho d \rho d \theta$, whereas the gradient writes $\nabla v=\partial_{\rho} v \boldsymbol{e}_{\rho}+$ $\rho^{-1} \partial_{\theta} v \boldsymbol{e}_{\theta}$. Because $\mathcal{O}$ is an interior domain, observe that one has $G=\mathbb{S}^{1}$ and $\partial \Gamma=\emptyset$. For the local edge problem at hand, since one is looking for $\Psi_{\mathrm{e}}=\rho^{\lambda_{\mathrm{e}}} \psi(\theta)$ in $H_{l o c}^{1}(\Gamma)$, one finds that a necessary and sufficient condition on the exponent $\lambda_{e}$ is that $\Re\left(\lambda_{e}\right)>$ 0 ; and moreover that

$$
\tau_{\mathrm{e}}(\xi):=\min _{\lambda_{\mathrm{e}} \text { st. } \Re\left(\lambda_{\mathrm{e}}\right)>0} \lambda_{\mathrm{e}},
$$

where $\Psi_{\mathrm{e}} \neq 0$ is a (non-smooth) function governed by $\operatorname{div}\left(\xi \nabla \Psi_{\mathrm{e}}\right)=0$ in $\Gamma$. The coefficient $\xi$ being independent of $\rho$, it is equivalent to finding solutions to the eigenproblem

$$
\left\{\begin{array}{l}
\text { Find } \psi \in H^{1}\left(\mathbb{S}^{1}\right) \backslash\{0\}, \nu \in \mathbb{C} \text { such that } \\
\int_{\mathbb{S}^{1}} \xi \partial_{\theta} \psi \partial_{\theta} \overline{\psi^{\prime}} d \theta=\nu \int_{\mathbb{S}^{1}} \xi \psi \overline{\psi^{\prime}} d \theta, \forall \psi^{\prime} \in H^{1}\left(\mathbb{S}^{1}\right),
\end{array}\right.
$$

with the relation $\nu=\lambda_{\mathrm{e}}^{2}$. The spectral theorem can be applied under the assumptions of section 2.1 for $\xi=\mu$ and $\xi=\varepsilon_{\sigma}$. As previously, $\psi=1$ and $\nu=0$ is an eigenpair of (B.7), leading to $\Psi_{\mathrm{e}}=1$, and one concludes again that no singular behavior is associated with $\nu=0$. So, using the Poincaré inequality in $H_{z m v}^{1}\left(\mathbb{S}^{1}\right)$, there exists $c_{P}>0$ such that, for all eigenfunctions $\psi$ related to a non-zero eigenvalue, the bound (B.4) holds, and one also recovers (B.2)-(B.3), with $\nabla_{\varsigma}$ replaced by $\partial_{\theta} \psi$, resp. $d \varsigma$ by $d \theta$. Then:

- If $\xi=\mu$ (real-valued coefficient case), one derives again the lower bound (B.5) on $\nu$. Due to the condition $\Re\left(\lambda_{\mathrm{e}}\right)>0$, the only admissible relation is $\lambda_{\mathrm{e}}=\sqrt{\nu}$. Hence $\lambda_{\mathrm{e}}>0$ for all non-zero eigenvalues $\nu$, which yields $\tau_{\mathrm{e}}(\mu)>0$. More precisely, one gets the lower bound

$$
\tau_{\mathrm{e}}(\mu) \geq c_{P}\left(\frac{\mu_{\min }}{\mu_{\max }}\right)^{1 / 2} .
$$

- If $\xi=\varepsilon_{\sigma}$ (complex-valued coefficient case), one has the lower bound (B.6) for $\nu_{R}$. And, according to (B.3), $\nu_{I}$ can take positive or negative values. Due to the condition $\Re\left(\lambda_{\mathrm{e}}\right)>0$ and because $\nu_{R}>0$, one has $\lambda_{\mathrm{e}}=\sqrt{\nu}$. Writing $\nu:=\nu_{R}+\imath \nu_{I}=\rho_{\nu} \exp \left(\imath \theta_{\nu}\right)$, with $\rho_{\nu}>0$ and $\theta_{\nu} \in\left(-\frac{\pi}{2}, \frac{\pi}{2}\right)$, one has $\lambda_{\mathrm{e}}=\rho_{\nu}^{1 / 2} \exp \left(\imath \theta_{\nu} / 2\right)$, so that $\Re(\sqrt{\nu}) \geq 2^{-1 / 2} \rho_{\nu}^{1 / 2}$. One may check that

$$
\Re(\sqrt{\nu}) \geq 2^{-1 / 2}\left(\frac{\left(\int_{\mathbb{S}^{1}} \xi_{R}\left|\partial_{\theta} \psi\right|^{2} d \theta\right)^{2}+\left(\int_{\mathbb{S}^{1}} \xi_{I}\left|\partial_{\theta} \psi\right|^{2} d \theta\right)^{2}}{\left(\int_{\mathbb{S}^{1}} \xi_{R}|\psi|^{2} d \theta\right)^{2}+\left(\int_{\mathbb{S}^{1}} \xi_{I}|\psi|^{2} d \theta\right)^{2}}\right)^{1 / 4} .
$$

Hence, one gets the lower bound

$$
\tau_{\mathbf{e}}\left(\varepsilon_{\sigma}\right) \geq 2^{-1 / 2} c_{P}\left(\frac{\varepsilon_{\min }^{2} \omega^{2}+\sigma_{\min }^{2}}{\varepsilon_{\max }^{2} \omega^{2}+\sigma_{\max }^{2}}\right)^{1 / 4} .
$$

B.2. Boundary domain. We consider now that the domain $\mathcal{O}$ on which a local problem is defined is a boundary domain, for which $\partial \mathcal{O} \cap \partial \Omega \neq \emptyset$. The main difference with the interior domain case is that the local problems now come with a (homogeneous) boundary condition: Dirichlet boundary condition for $p_{0}$, resp. Neumann 
boundary condition for $q_{0}$. On the other hand, the theory is quite similar to the one of $\S B .1$. As in the interior domain case, one must consider edge problems, and corner problems. Below, we suppose explicitly that $\mathcal{O} \cap \Sigma \neq \emptyset$.

For the corner problem, one looks for homogeneous solutions that belong to $S_{c}^{\lambda}(\Gamma)$, but now $\partial \Gamma \neq \emptyset$, and $G=\Gamma \cap \mathbb{S}^{2}$ is a strict subset of $\mathbb{S}^{2}$. Then, one solves an eigenproblem like (B.1), with the relation $\nu=\lambda_{c}\left(\lambda_{c}+1\right)$, now set in the function space $H_{0}^{1}(G)$ (Dirichlet boundary condition), resp. in $H_{z m v}^{1}(G)$ (Neumann boundary condition). The main observation for the corner problem set in a boundary domain is that, since there holds a Poincaré inequality in both function spaces, one may still apply the previous analysis (interior domain), to draw the conclusions. Namely, one finds that

$$
\tau_{\mathrm{c}}(\mu)>1 / 2, \quad \tau_{\mathrm{c}}\left(\varepsilon_{\sigma}\right)>1 / 2,
$$

independently of the values of the coefficients.

For the edge problem, one looks for homogeneous solutions that belong to $S_{\mathrm{e}}^{\lambda}(\Gamma)$, where $\partial \Gamma \neq \emptyset$, and $G$ is a strict subset of $\mathbb{S}^{1}$. One solves an eigenproblem like (B.7), with the relation $\nu=\lambda_{\mathrm{e}}^{2}$, set in the function space $H_{0}^{1}(G)$ (Dirichlet boundary condition), resp. in $H_{z m v}^{1}(G)$ (Neumann boundary condition). Since there holds a Poincaré inequality in both function spaces, one may again apply the previous analysis (interior domain), to draw the conclusions. One finds the lower bounds:

$$
\tau_{\mathrm{e}}(\mu) \geq c_{P, \text { Neu }}\left(\frac{\mu_{\min }}{\mu_{\max }}\right)^{1 / 2}, \quad \tau_{\mathrm{e}}\left(\varepsilon_{\sigma}\right) \geq 2^{-1 / 2} c_{P, D i r}\left(\frac{\varepsilon_{\min }^{2} \omega^{2}+\sigma_{\min }^{2}}{\varepsilon_{\max }^{2} \omega^{2}+\sigma_{\max }^{2}}\right)^{1 / 4} .
$$

Finally, if there is no interface in $\mathcal{O}$, ie. $\mathcal{O} \cap \Sigma=\emptyset$, one simply considers that the coefficient $\xi$ is constant on $\mathcal{O}$. In this case the value of $\tau_{\mathrm{c}}, \tau_{\mathrm{e}} \in\left(\frac{1}{2}, 1\right]$ is determined by the geometry of the boundary. Precisely, if $\mathcal{O}$ is defined as the intersection of $\Omega$ with a ball, one finds that $\tau_{\mathrm{c}}<1$ or $\tau_{\mathrm{e}}<1$ if, and only if, $\mathcal{O}$ is not convex.

B.3. Behavior of the best exponent. From the previous studies, we conclude that one derives the actual value of the best regularity exponent by taking $\tau_{(\varepsilon, \mu, \sigma)}^{o p t}:=$ $\min \left(1, \min _{\mathrm{e}} \tau_{\mathrm{e}}, \min _{\mathrm{c}} \tau_{\mathrm{c}}\right)$. In particular, one may compute numerically the value of $\tau_{(\varepsilon, \mu, \sigma)}^{\text {opt }}$.

As for the global approach (see §6.5), let us study what happens when $\Theta$ is not reduced to a singleton. Again, let us consider that all its elements $(\varepsilon, \mu, \sigma)$ are such that $\varepsilon, \sigma, \mu$ are piecewise-constant on a fixed partition. We see that if condition

$$
\sup _{(\varepsilon, \mu, \sigma) \in \Theta} \frac{\mu_{\max }}{\mu_{\min }}+\sup _{(\varepsilon, \mu, \sigma) \in \Theta}\left(\frac{\varepsilon_{\max }^{2} \omega^{2}+\sigma_{\max }^{2}}{\varepsilon_{\min }^{2} \omega^{2}+\sigma_{\min }^{2}}\right)^{1 / 2}<\infty
$$

holds, then $\inf _{(\varepsilon, \mu, \sigma) \in \Theta} \tau_{(\varepsilon, \mu, \sigma)}^{o p t}>0$. 